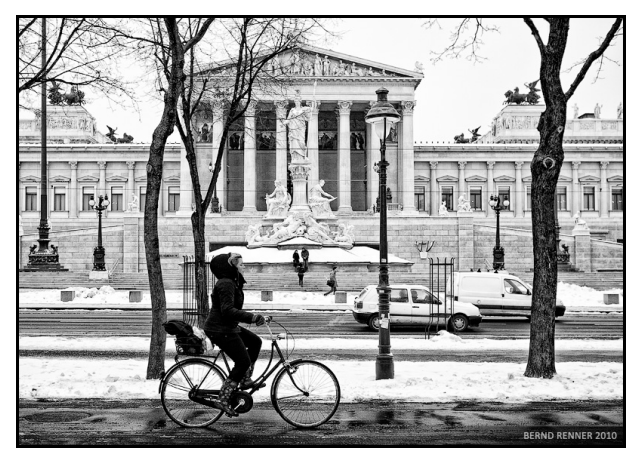

$13^{\text {th }}$ Vienna Conference on Instrumentation 11-15 February 2013

\title{
Performance, Radiation Damage Effects and Upgrade of the LHCb Vertex Locator
}

Stefano de Capua

on behalf of the LHCb VELO group

\section{MANCHESTER 1824}




\section{Overview}

口 Introduction to $\mathrm{LHCb}$

- The LHCb Vertex Locator and its performance.

- Radiation damage effects.

口 Upgrade.

- H. Schindler:

The LHCb detector upgrade

- M. Tobin:

The LHCb Silicon Tracker

- F. Dettori:

Performance of $\mathrm{LHCl}$

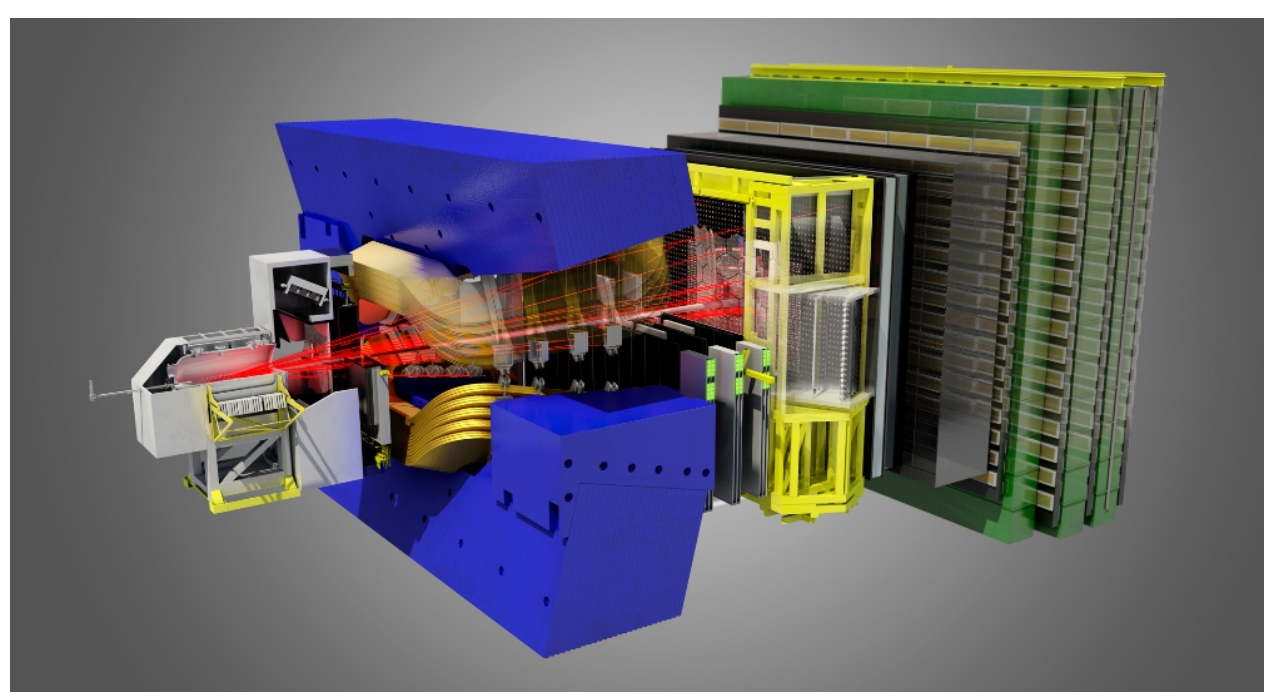




\section{The LHCb detector}

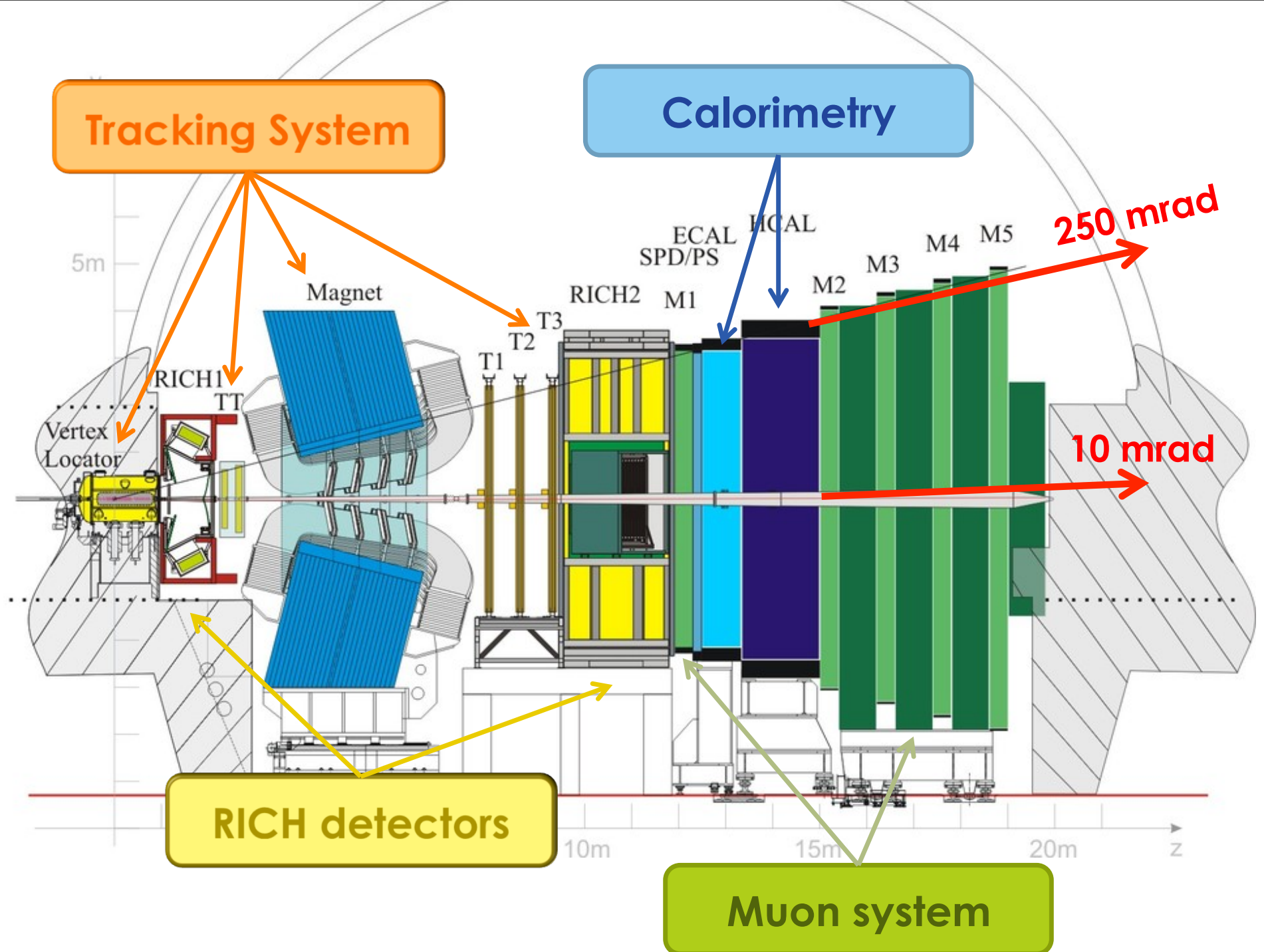




\section{The LHCb detector}

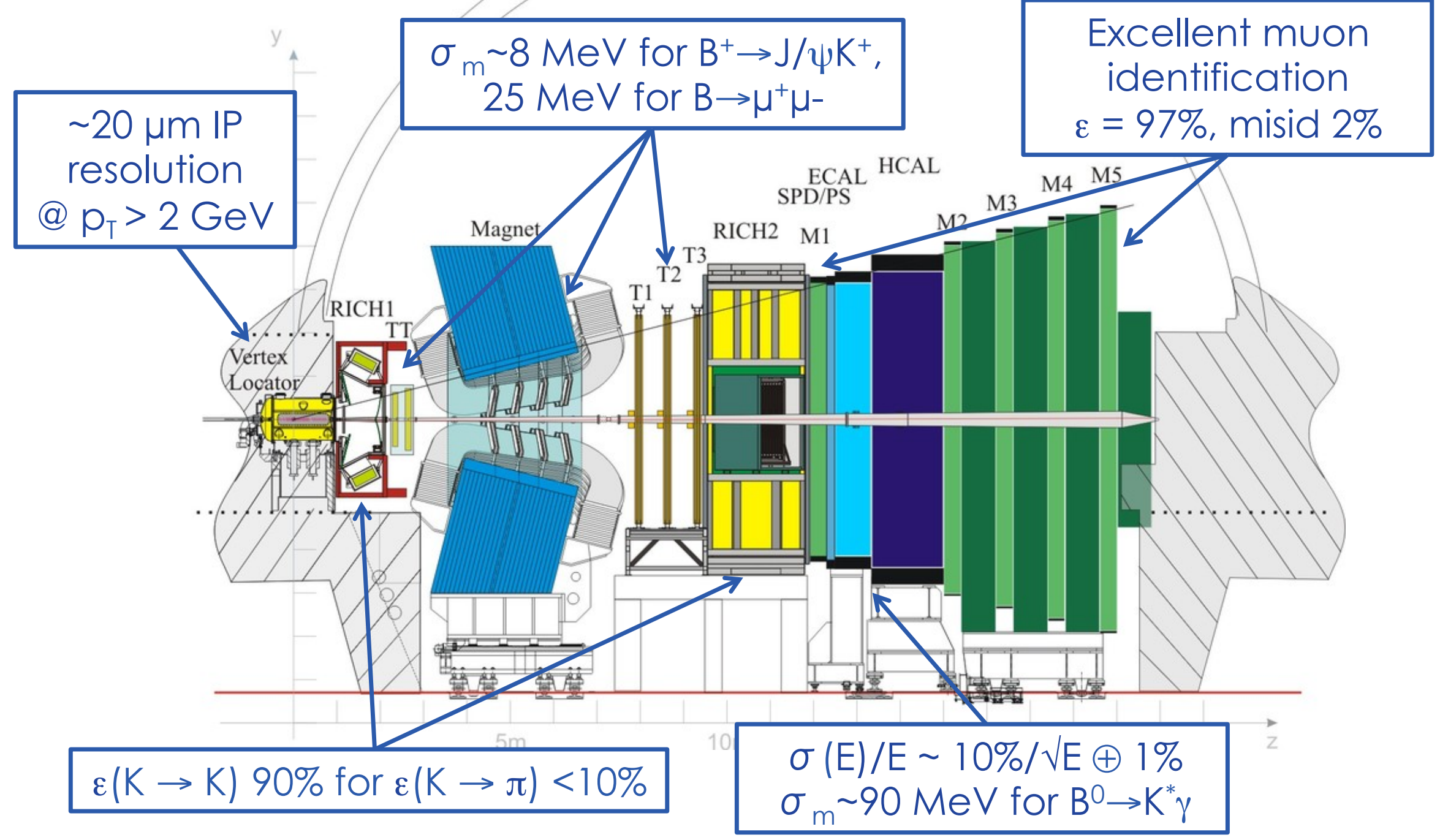




\section{The VELO detector}
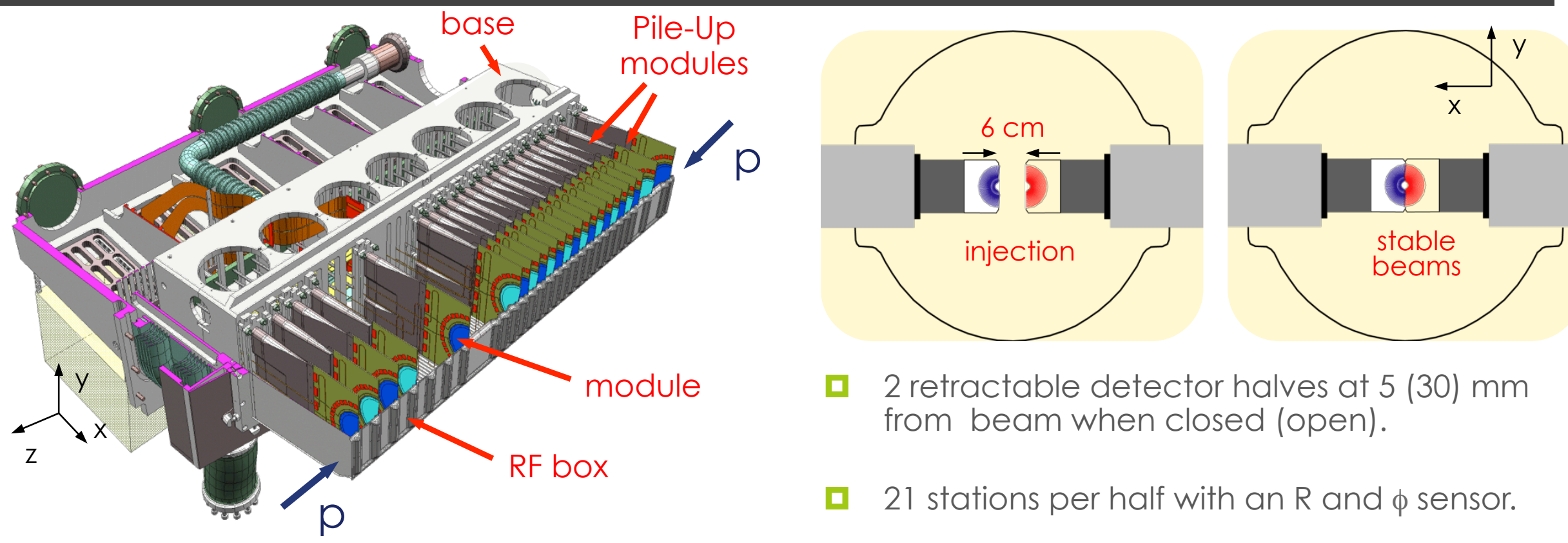

- 2 retractable detector halves at 5 (30) mm from beam when closed (open).

- 21 stations per half with an R and $\phi$ sensor.

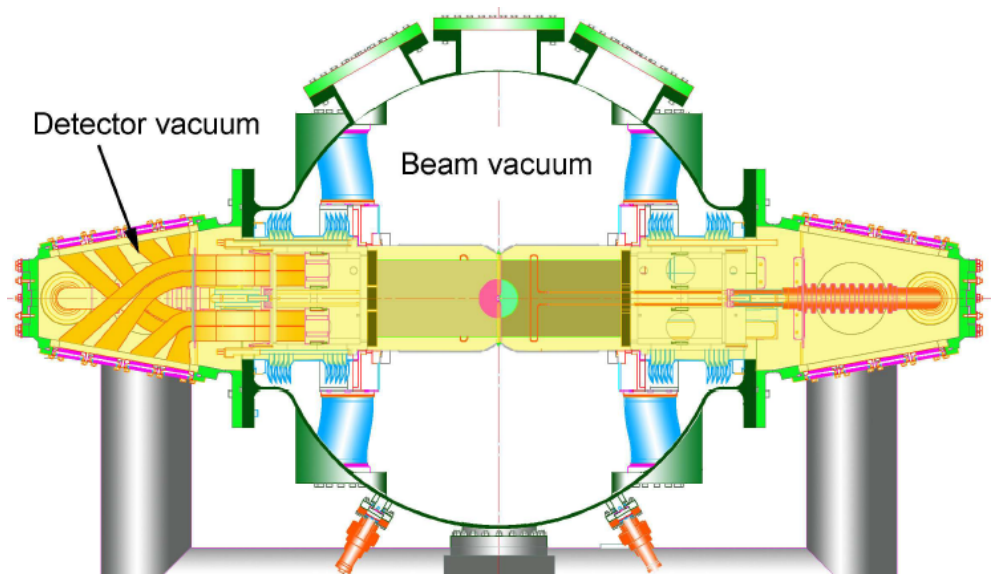

ㅁ 2 Pile-Up stations per half (trigger).

- Operates in secondary vacuum.

- $300 \mu \mathrm{m}$ foil separates detector from beam vacuum.

- $\mathrm{CO}_{2}$ cooling system (Operates @ -30 ${ }^{\circ} \mathrm{C}$, Sensors@-10 $\mathrm{C}$. 


\section{The VELO sensors}

- $300 \mu \mathrm{m}$ n+-on-n sensors (Micron). - One module with n+-on-p (Micron)

- R-sensors:

- 45 degree quadrants

D Pitch $=40-101.6 \mu \mathrm{m}$.

( $\Phi$-sensors:

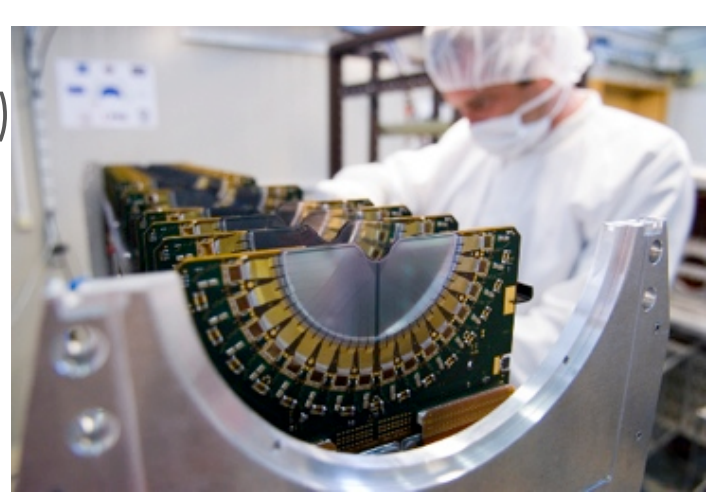

- 2 regions (short/long strips)

口 Pitch $=35.5-96.6 \mu \mathrm{m}$

- 2048 strips / sensor

口 172,032 strips in total

- Double metal layer for signal routing.

- First active strip @ $\mathrm{R} \approx 8 \mathrm{~mm}$.

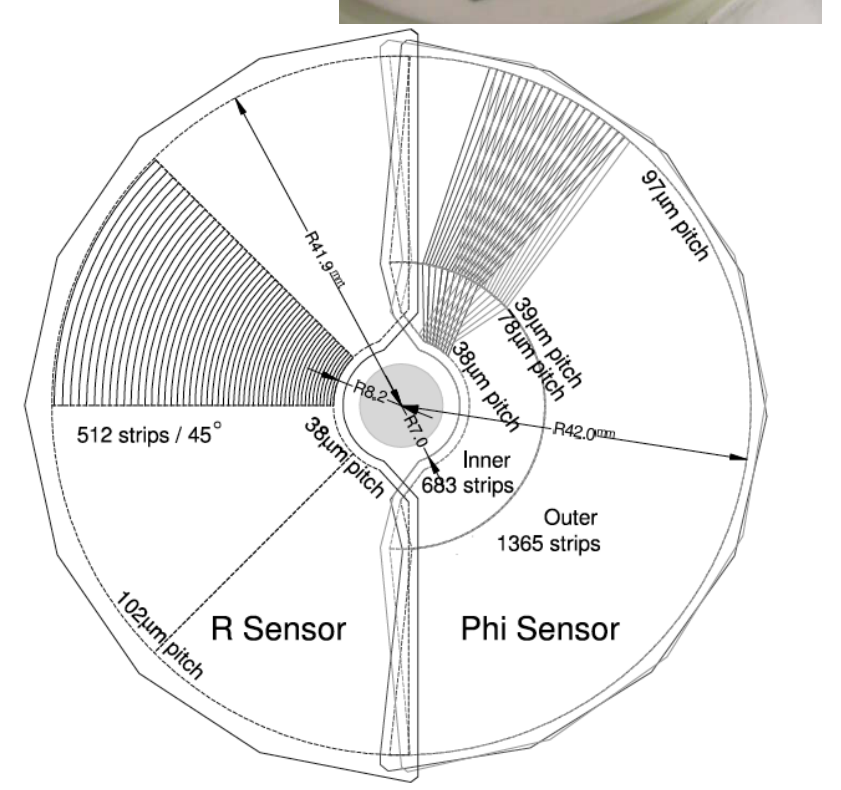




\section{Alignment}

- VELO halves centered around beam at each fill, when beams declared stable.

- Beam position determined from vertex reconstruction with tracks in right or left half.

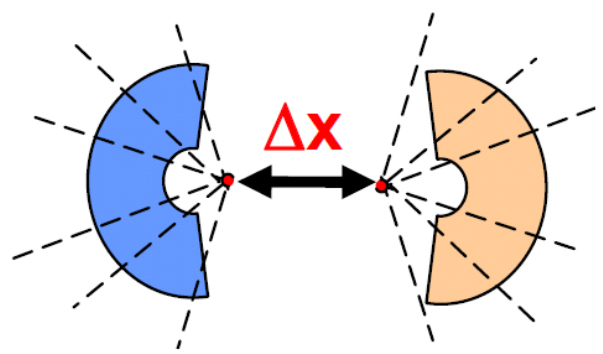

- Misalignment from distance between the two reconstructed vertices.

- Fully automated procedure ( 210 s).

a Stable within $\pm 5 \mu \mathrm{m}(\mathrm{x})$.
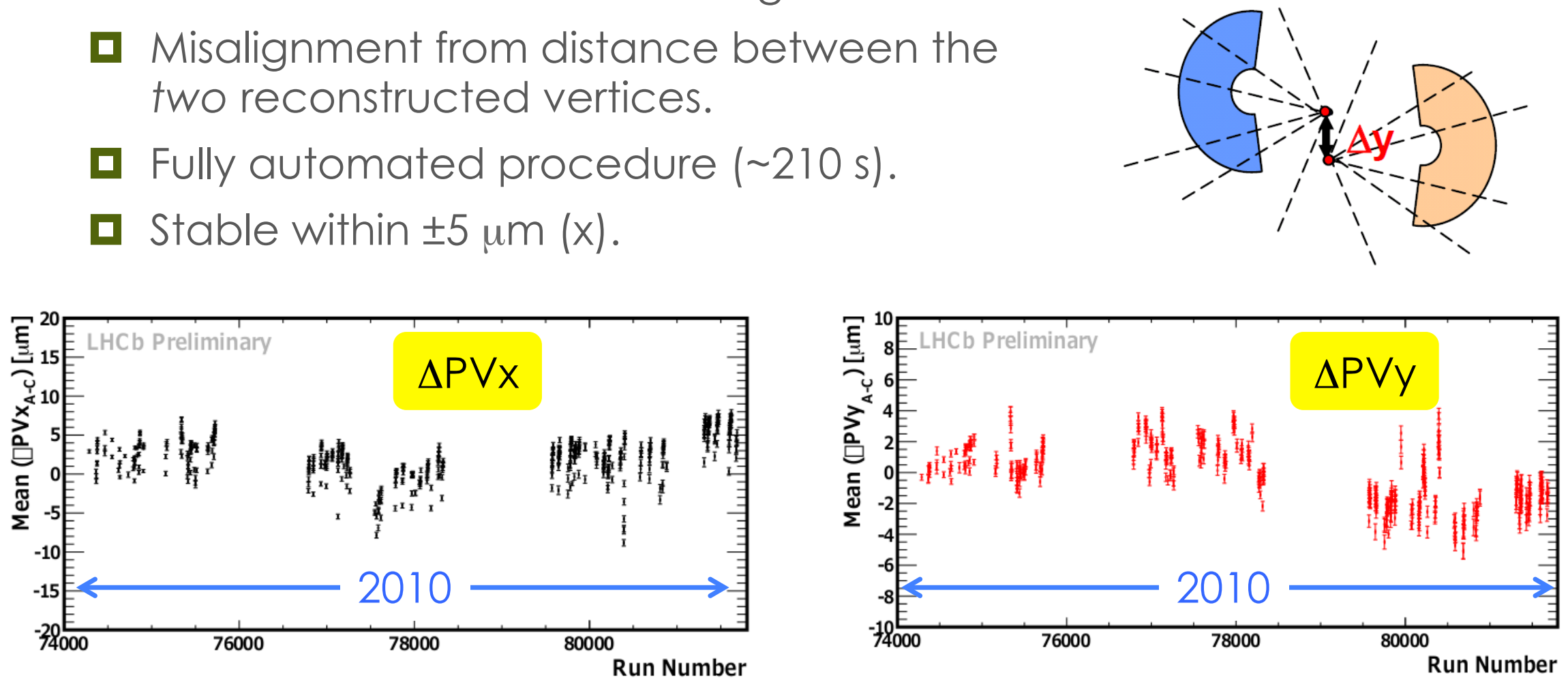


\section{Performance}
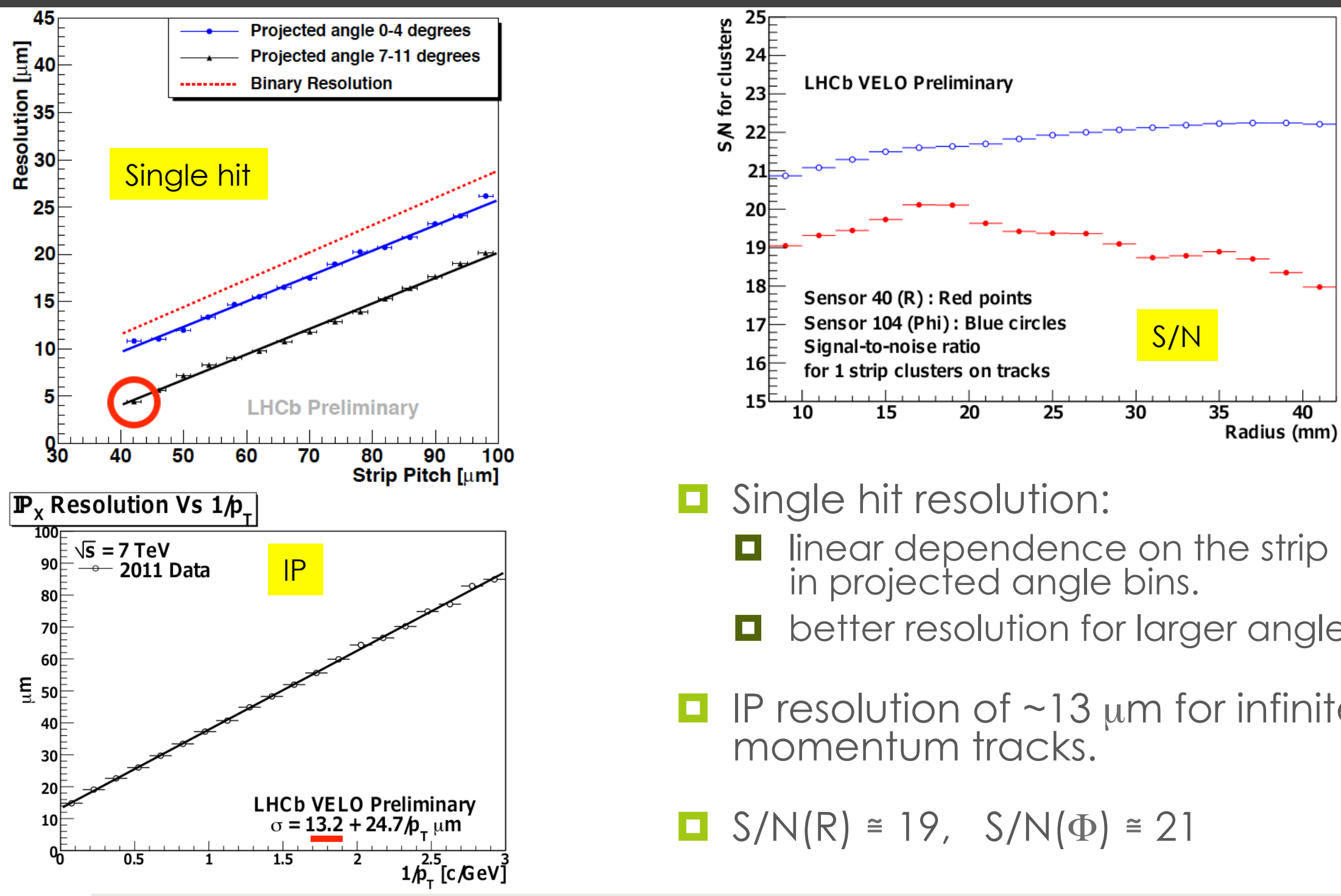

- Single hit resolution:

- linear dependence on the strip pitch in projected angle bins.

- better resolution for larger angles.

- IP resolution of $\sim 13 \mu \mathrm{m}$ for infinite momentum tracks.

$S / N(R) \cong 19, S / N(\Phi) \cong 21$ 


\section{Performance}

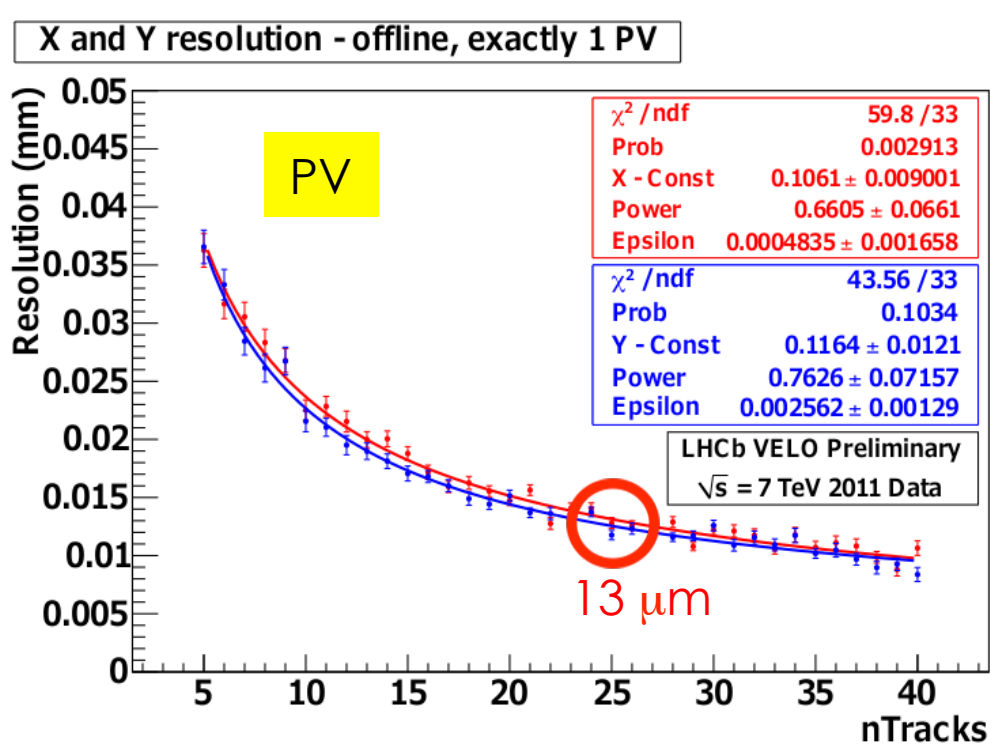

$\omega$

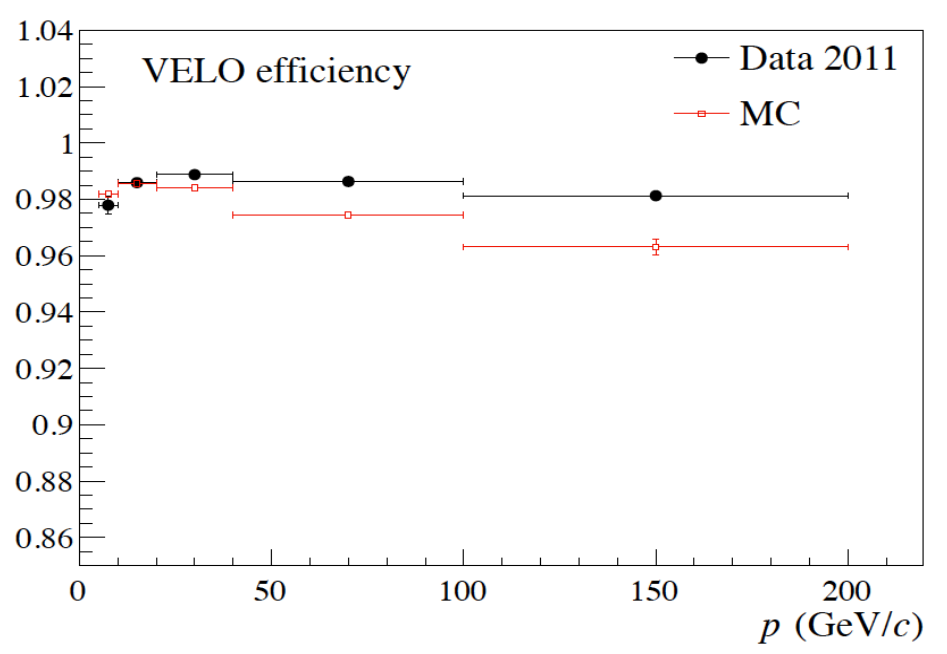

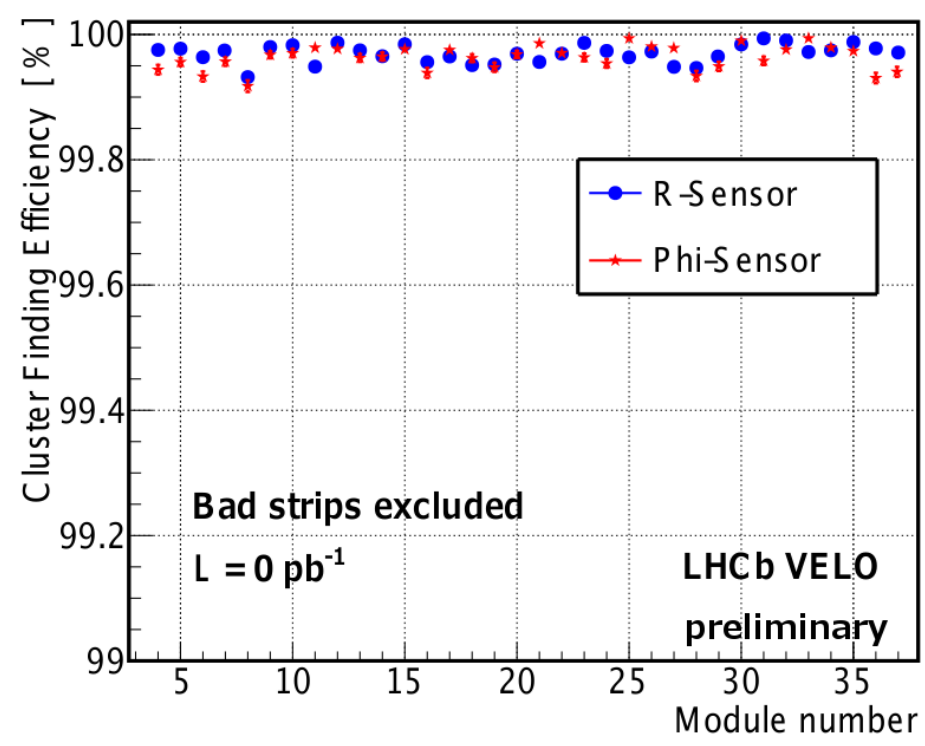

- Excellent cluster finding efficiency:

口 CFE $=99.51 \pm 0.02 \%$ [all channels]

a $\mathrm{CFE}=99.98 \pm 0.02 \%$ [bad strips excl, $0.77 \%$ ]

- PV resolution of $13 \mu \mathrm{m}$ for 25 tracks (typical case).

- Excellent tracking efficiency. 


\section{Radiation damage}

a High particle fluence (up to $5 \times 10^{13} 1 \mathrm{MeV} \mathrm{n}_{\text {eq }} / \mathrm{cm}^{2}$ ) due to proximity to the beams.

- Particle irradiation causes surface and bulk damages in the silicon $=>$ affects leakage current and effective doping concentration.

- 4 independent and complementary methods to monitor these effects:

- changes in currents vs. voltage (IV curve)

$\square$ changes in current vs. temperature (IT curve)

- changes in Full Depletion Voltage

口 changes in Cluster Finding Efficiency 


\section{Current vs Voltage/Temperature}

a Bulk current increases with fluence proportionally to the delivered luminosity.

- All sensors at nominal bias (150 V) and at $-8^{\circ} \mathrm{C}$.
- Typically $1.9 \mu \mathrm{A}$ per $100 \mathrm{pb}^{-1}$.

- Good agreement with expectation from fluence.

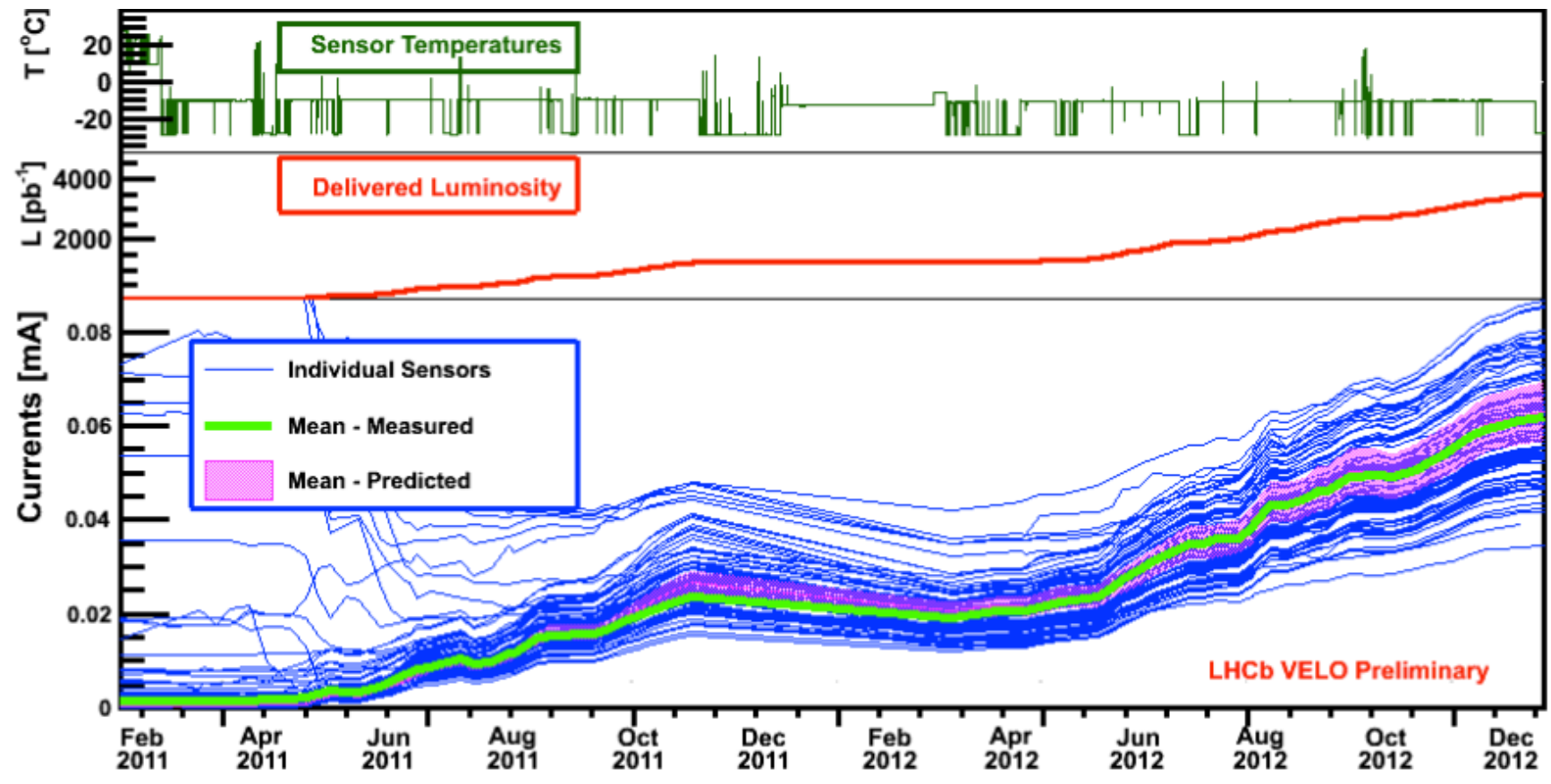




\section{Effective Depletion Voltage}

- During assembly measured by comparing capacitance to bias voltage across the sensor. Not possible after installation

=> dedicated runs every 3-4 months.

- Method: extrapolate tracks to test sensor (every $5^{\text {th }}$ module) and determine amount of charge collected.

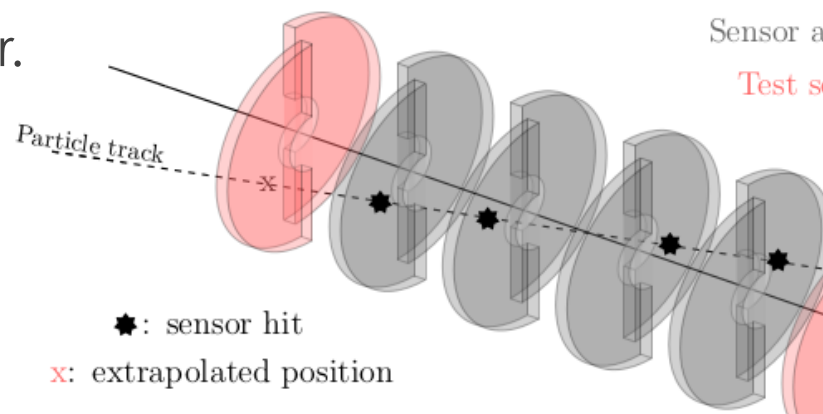

Sensor at $150 \mathrm{~V}$

Test sensor

Voltage of test sensor varied between 0-150 V. At each bias voltage the most probable value (MPV) of ADC counts distribution is determined. Effective Depletion Voltage (EDV) defined as the voltage at which the MPV is $80 \%$ of the plateau.
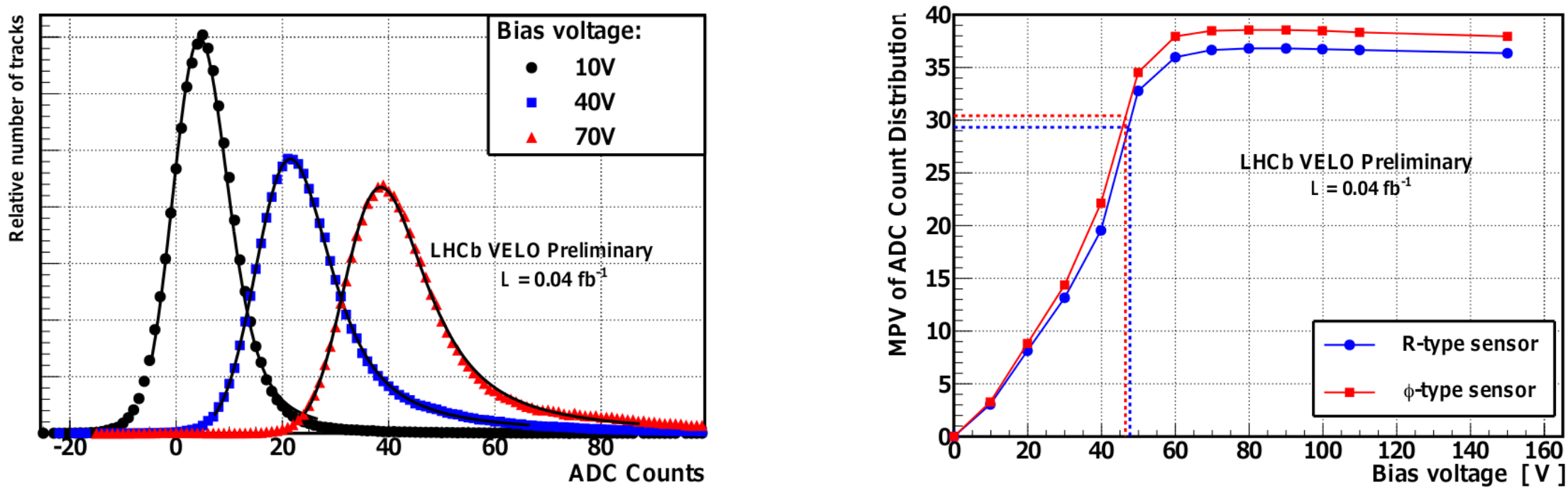


\section{Effective Depletion Voltage}

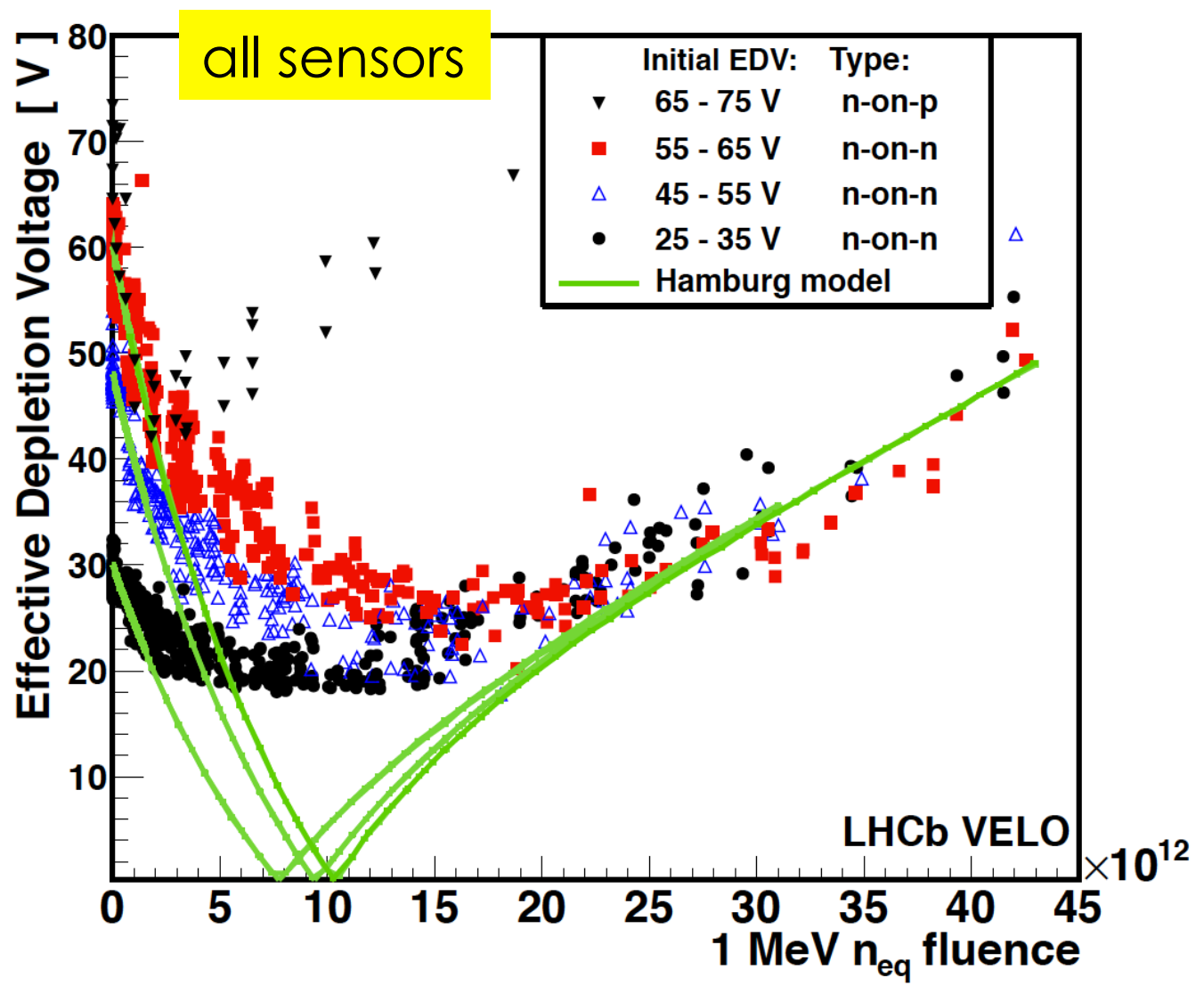

Minimum EDV 18 $\vee$ before type inversion, which occurs at

$\sim 15 \times 10^{12} 1 \mathrm{MeV} \mathrm{n}_{\text {eq. }}$.

Subsequent increase is $\sim$ linear.

EDV initially decreases with fluence for all radius. After type inversion, EDV increases with fluence. Type inversion starts at inner radius.

Sensors are operated at $150 \mathrm{~V}$ and can be operated up to $500 \mathrm{~V}$.

Good agreement with Hamburg model at low and high fluences. Discrepancy around type inversion because of finite charge collection time. 


\section{2nd metal layer}

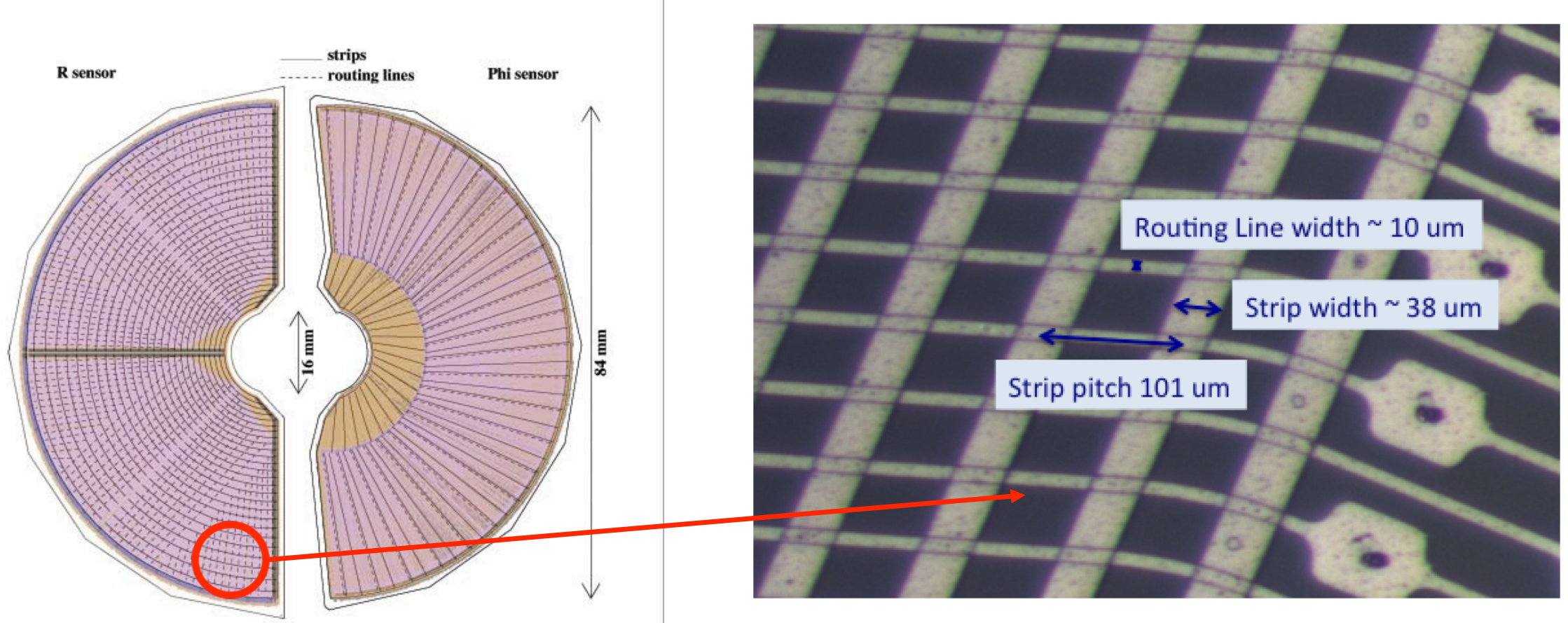

- $\quad 1^{\text {st }}$ metal layer capacitively couples to strips. $2^{\text {nd }}$ metal layer carries signal out to electronics.

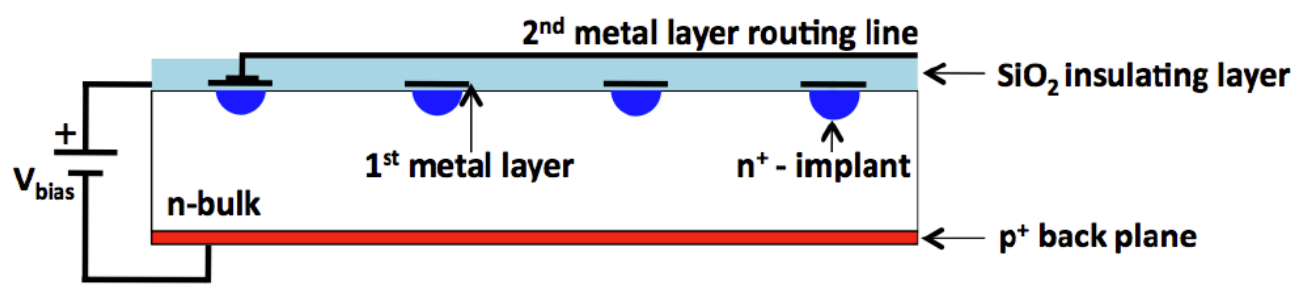

- Routing lines are perpendicular to strips in R-sensors and parallel to strips in $\Phi$-sensors. 


\section{Routing lines}

口 Light blue: ${ }^{\text {st }}$ metal layer.

Dark blue: $2^{\text {nd }}$ metal layer.

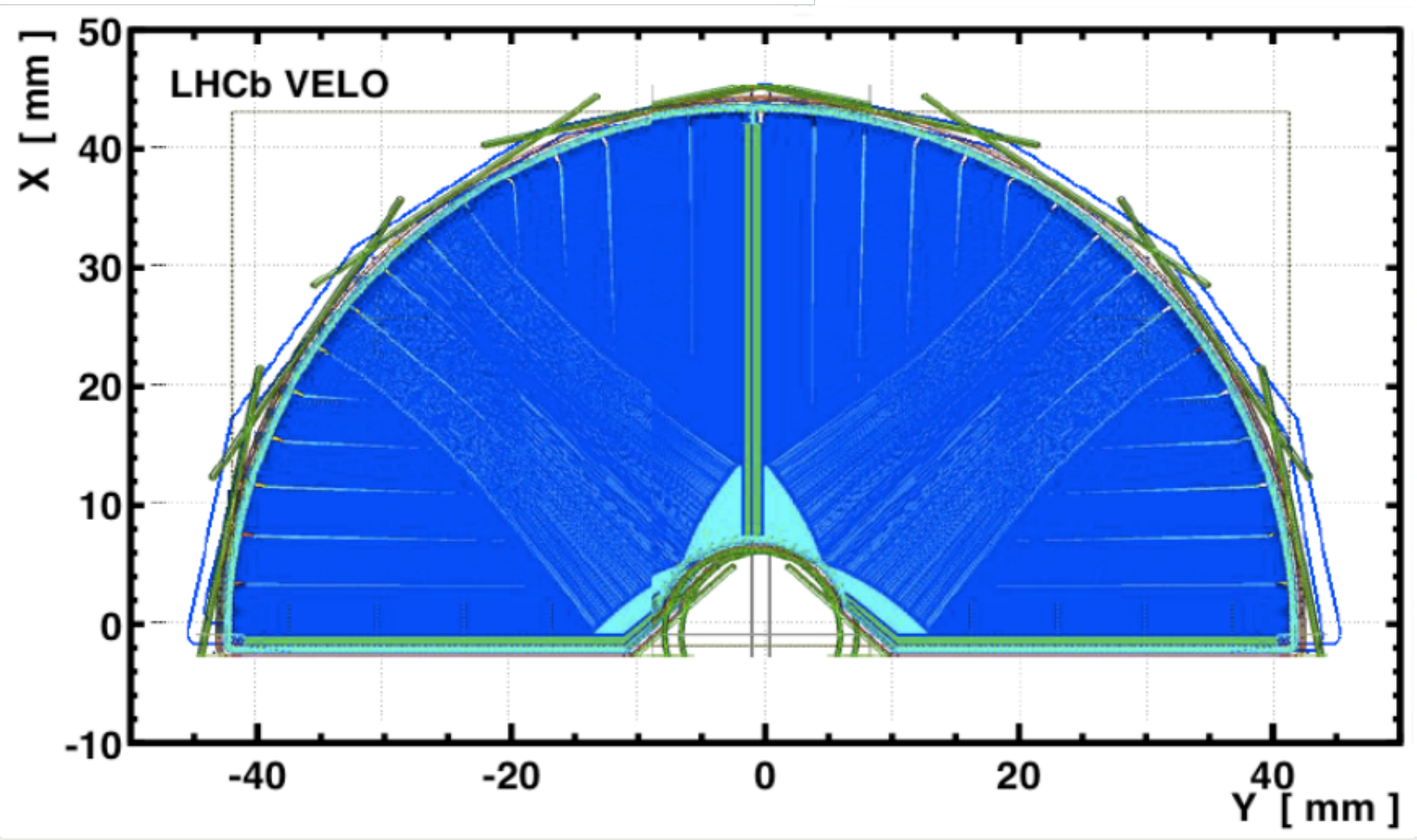




\section{Cluster Finding Efficiency}

- Clusters defined as one or more adjacent strips with charge over threshold.

- Finding and reconstructing clusters efficiently is a key aspect for physics performance.

- Measured with dedicated scans as well as during physics runs.

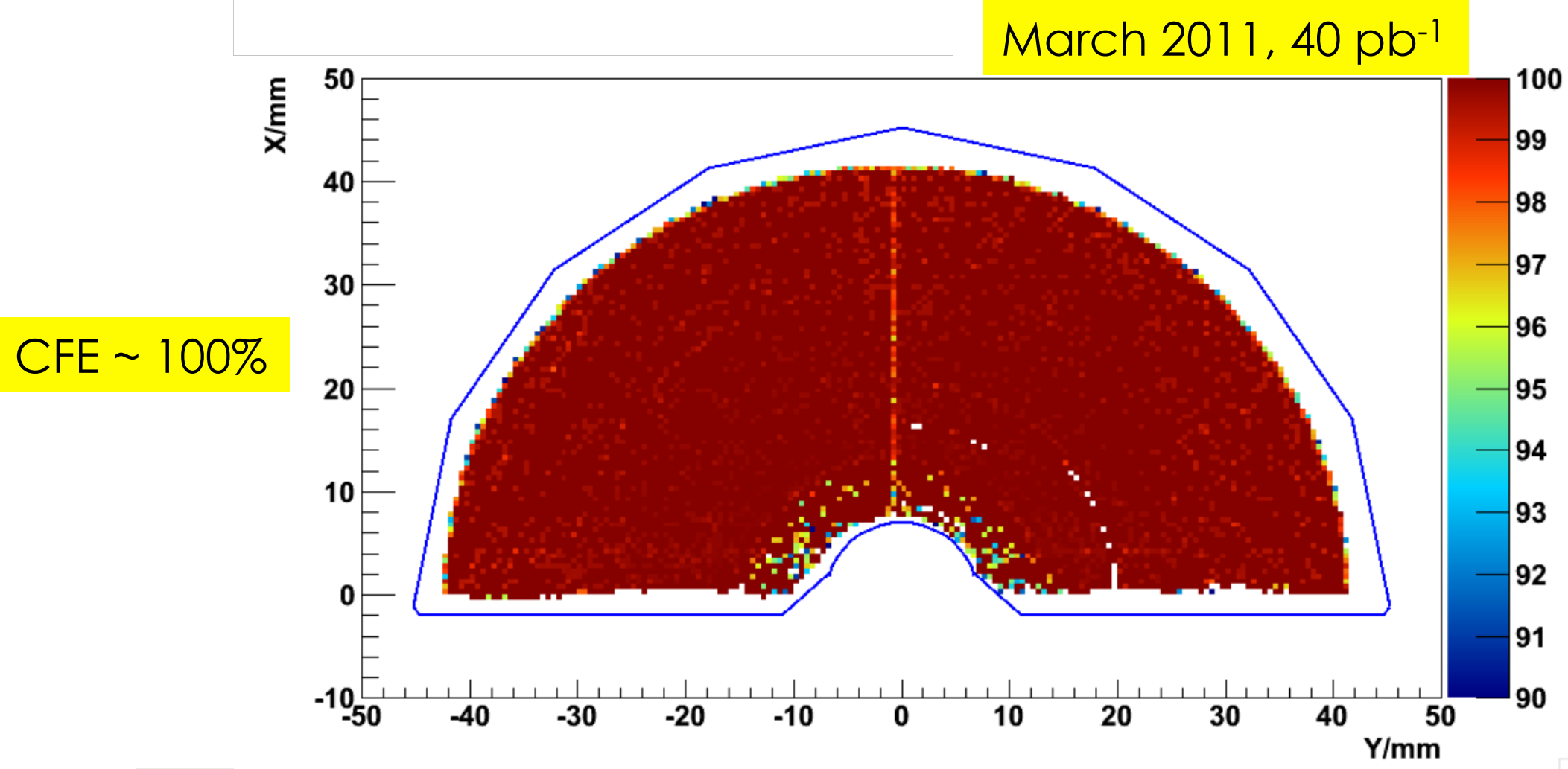




\section{Cluster Finding Efficiency}

- Cluster Finding Efficiency drops with fluence, especially in outer radial regions.

口 Higher efficiency where no routing lines !

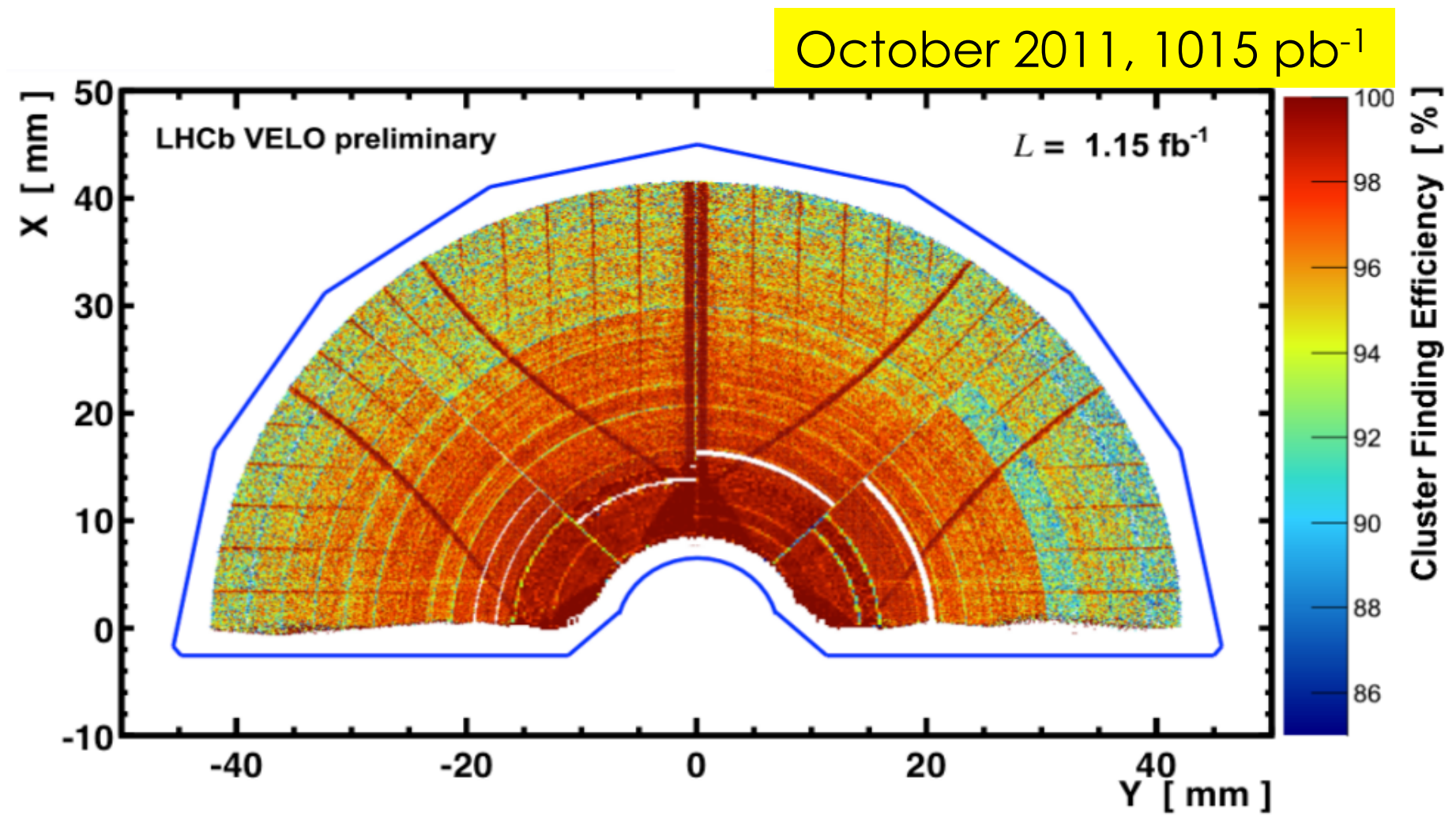




\section{Strip distance dependence}

Drop of CFE in regions far away from strips and close to routing lines.

- No effect on tracking efficiency observed.
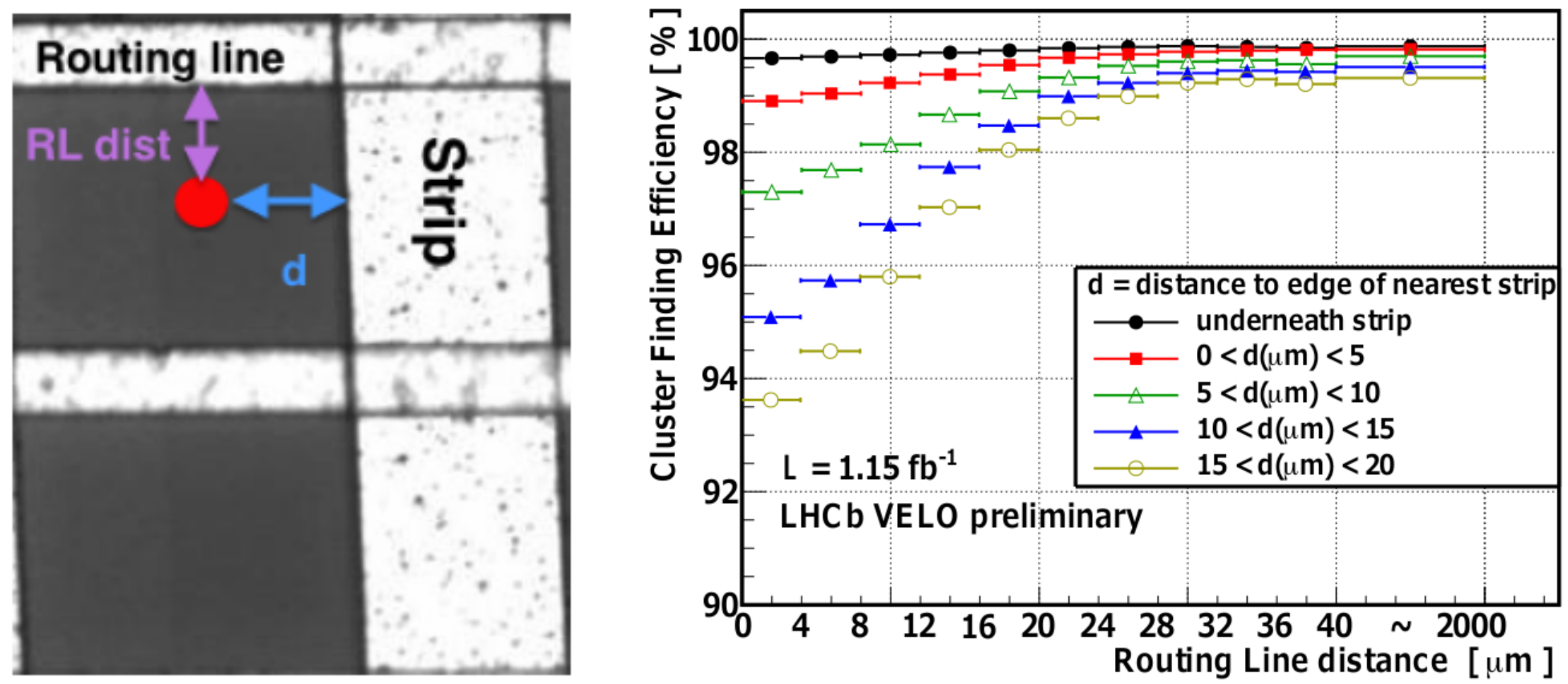


\section{VELO Upgrade}

- Why upgrade LHCb? Current limitation due to hardware trigger $=>$ no gain for hadronic channels from higher luminosity => move to a full software trigger!

口 Detector to be operated @ $40 \mathrm{MHz}$ and $2 \times 10^{33} \mathrm{~cm}^{-2} \mathrm{~s}^{-1}$

a huge data rates (>2 Tbit/s): new front-end electronics and sensors

口 radiation hard (radiation levels up to $5 \times 10^{15} 1 \mathrm{MeV} \mathrm{n}_{\mathrm{eq}} \mathrm{cm}^{-2}$ )

口 new cooling interface

口 improve performance

- Two options: Microstrips or pixels

口 $200 \mu \mathrm{m}$ n-on-p thick silicon

a high thermally conductive spine

Proposed strip detector layout

口 re-use of existing infrastructures (CO2, HV/LV, vacuum, motion)

口 new custom RF-foil

a dead material (at periphery for strips, i.e. out of acceptance)

口 pixel option uses a single flip chip assemblies per module
Proposed pixel detector layout

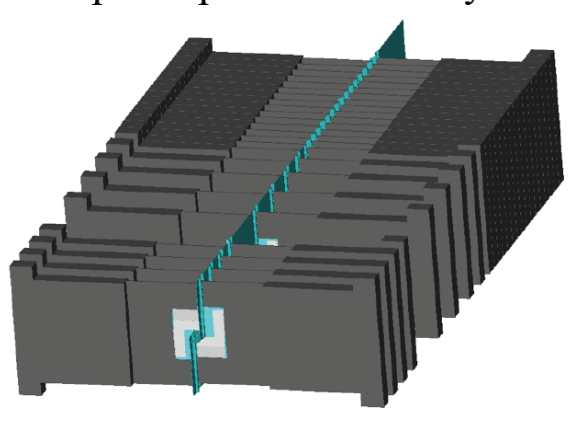




\section{Strips}

- Conservative option (R $\Phi$ geometry)

- Increase number of strips (20 chips $\times 128$ channels per sensor), reduce pitch (30 $\mathrm{mm}$ minimum) $=>$ keep occupancies $<0.6 \%$ at $10^{33} \mathrm{~cm}^{-2} \mathrm{~s}^{-1}$

- Sensitive area close to the edge: active @ $7 \mathrm{~mm}$ from the beam

- Sensor prototypes (Hamamatsu) being tested, sensor hybrid to be developed

- New ASIC chip under development: on-chip common mode subtraction, zero suppression and possibly clustering

- 1 ADC per strip
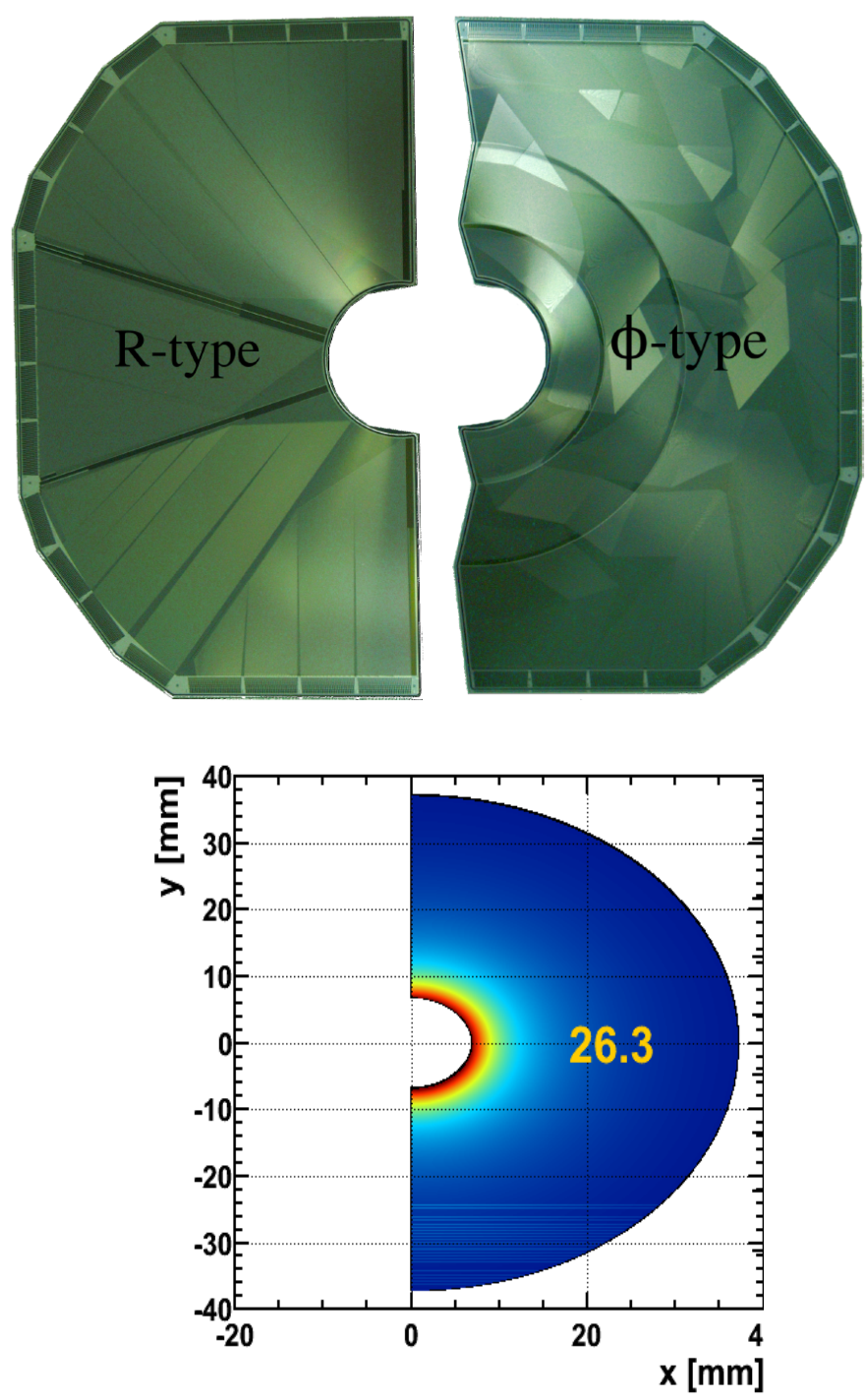


\section{Pixels}

- Based on VeloPix, successor of TimePix

- Silicon Planar Sensors 55x55 um pixel, $256 \times 256$ array

a Simultaneous measurement of timeover-threshold (TOT) and time-of-arrival (ToA)

=> ideal for time stamp and inter pixel positioning

- Requirements: peaking time $<25 \mathrm{ns,}$ timewalk<25 ns @1ke-

a Count rate up to $500 \mathrm{MHz} / \mathrm{chip}$

a Power consumption < $3 \mathrm{~W} /$ chip
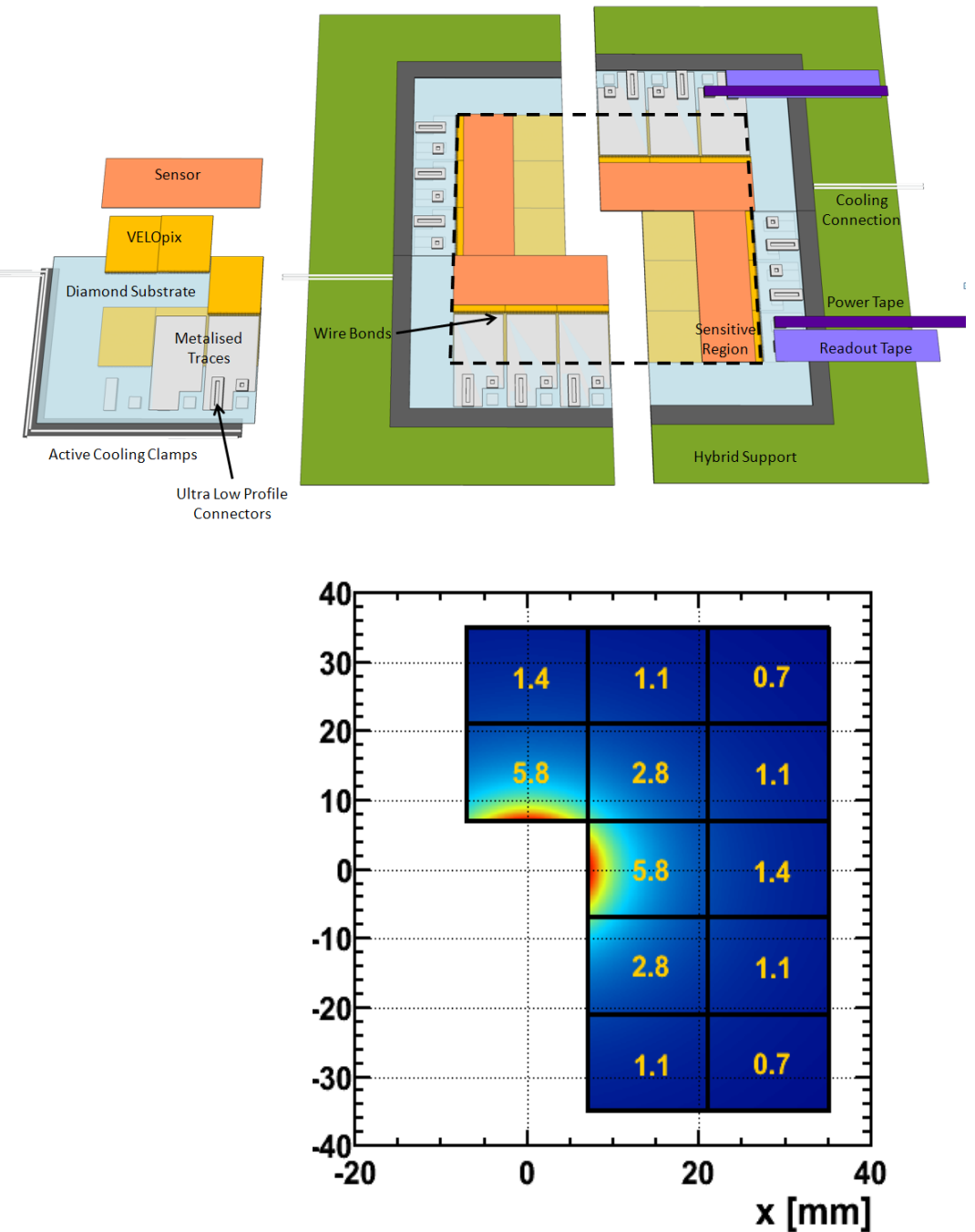


\section{Cooling interface}

- Three cooling options currently being pursued.

- TPG substrate/blocks

- Baseline and more conservative option

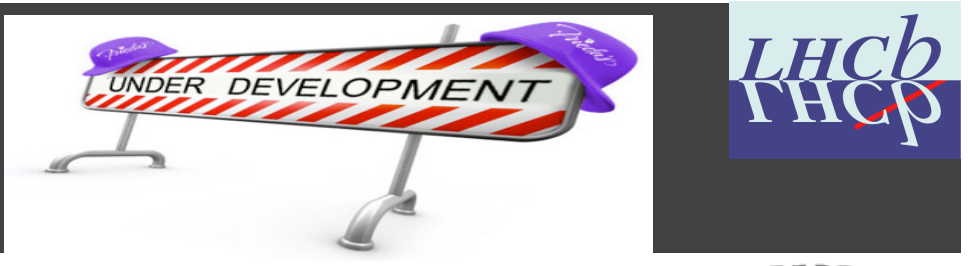

- - -

- Micro channels

- silicon substrate (no stress due to CTE)

- low material budget

- routing of channels customizable

- 60 bar pressure under chip

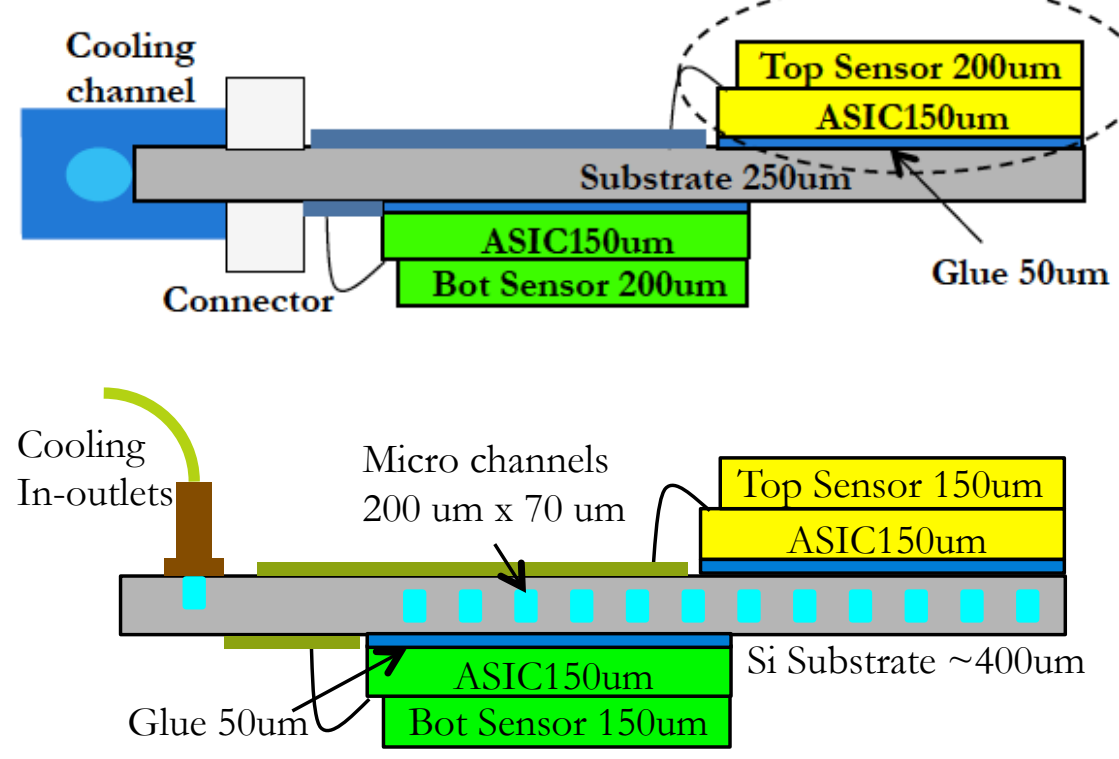

- C-foam and invar tubes

- low material budget

口 integrated cooling
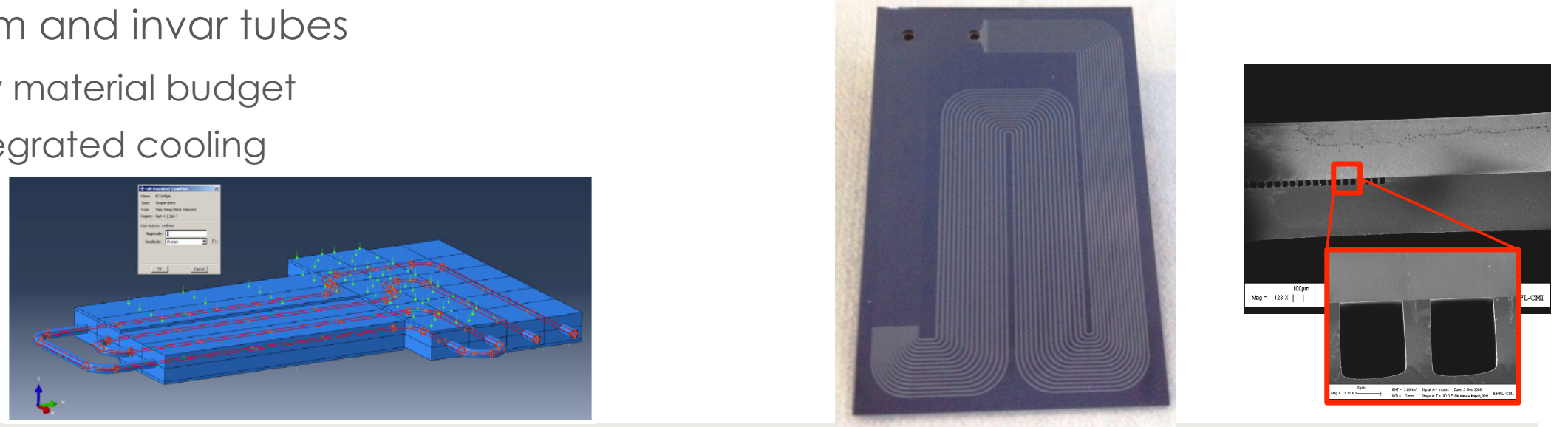


\section{Conclusion}

- The LHCb VELO has been operated smoothly during the physics data taking and has showed excellent performance.

- $S / N(R)=19, S / N(\Phi)=21$.

- Single hit resolution $=4 \mu \mathrm{m}$ at $40 \mu \mathrm{m}$ pitch.

口 Typical IP resolution $=13(12) \mu \mathrm{m}$ in $x(y)$.

- Typical PV resolution $=13$ (71) $\mu \mathrm{m}$ in $\mathrm{x}, \mathrm{y}(\mathrm{z})$.

- Excellent Cluster Finding and tracking efficiencies.

- Radiation damage effects have been studied.

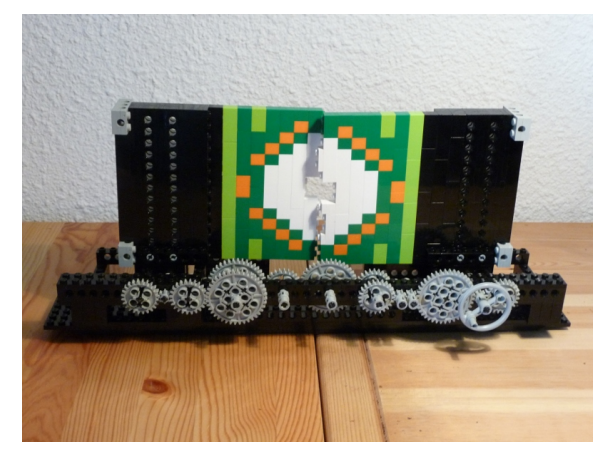

- Currents increase $\sim 1.9 \mu \mathrm{A}$ per 100/pb.

- Type inversion observed.

- Increase of Effective Depletion Voltage.

- $2^{\text {nd }}$ metal layer effect observed.

口 The detector will be upgraded in 2018 to cope with a $40 \mathrm{MHz}$ readout trigger.

- Two options under investigation: strips or pixels.

口 R\&D of cooling interface, module design, FE electronics. 


\section{Spares}




\section{LHCb operation}

LHCb Integrated Luminosity pp collisions 2010-2012

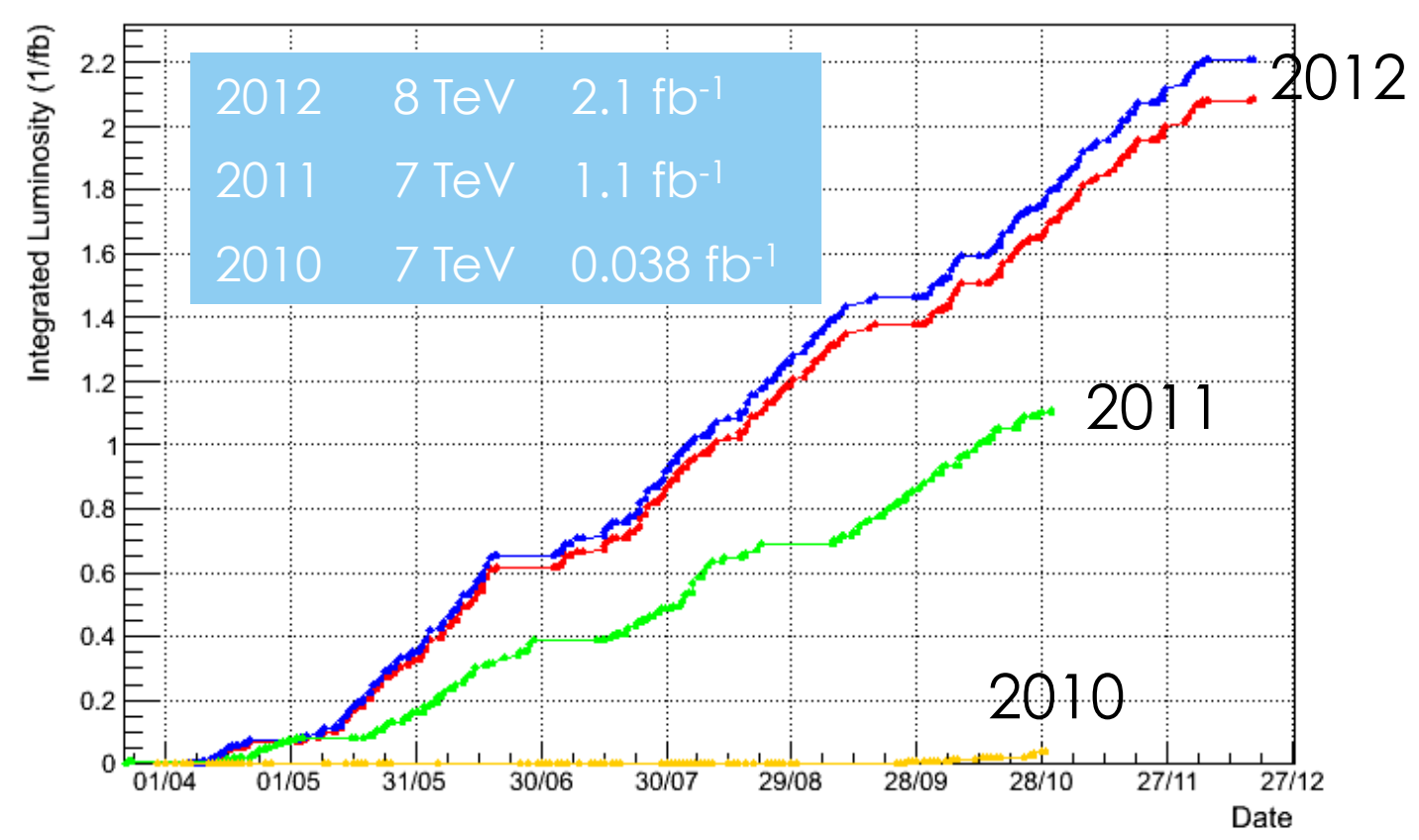

a Lumi-Leveling: semi-continuous (automatic) adjustment of offset of colliding beams allowing luminosity to be constant.

- 4 times more collisions per bunch crossing than in the design!
FULLY ON: 93.05 (\%) HV: $0.54(\%)$ VELO Safety: $0.85(\%)$ DAQ: $2.85(\%)$ DeadTime: $2.88(\%)$

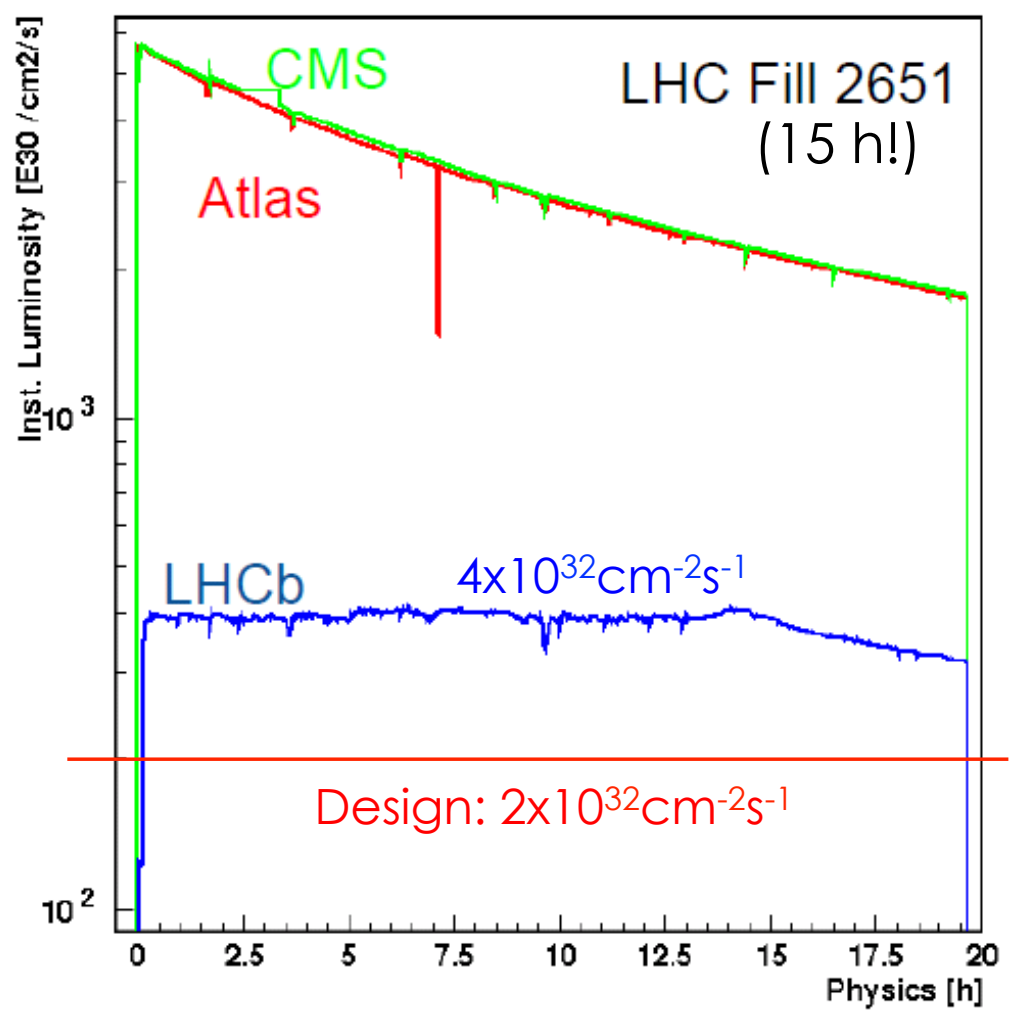




\section{Alignment}

ㅁ Crucial for hit/impact parameter/decay time resolution!

Starting point: survey before installation

- Relative R-sensor to $\Phi$-sensor position measured with accuracy of $3 \mu \mathrm{m}$ (translations in $\mathrm{x}, \mathrm{y}$ ) and 20 urad (rotations around $x, y$ )

- Relative module positions in each half measured with accuracy of $10 \mu \mathrm{m}$ (translations in $\mathrm{x}, \mathrm{y}$ )

- Position of two halves with accuracy of $100 \mu \mathrm{m}$ (transl.) and $100 \mu \mathrm{rad}$ (rotat.).

- Software alignment: 2 methods based on minimising the residuals btw tracks and hits.

1. Sensors, modules and halves alignment based on Millipede [NIM A596 (2008) 157 and 164].

2. Global $\chi^{2}$ minimisation based on Kalman track fit residuals [NIM A600 (2009) 471].

misalignment better than $4 \mu \mathrm{m}$
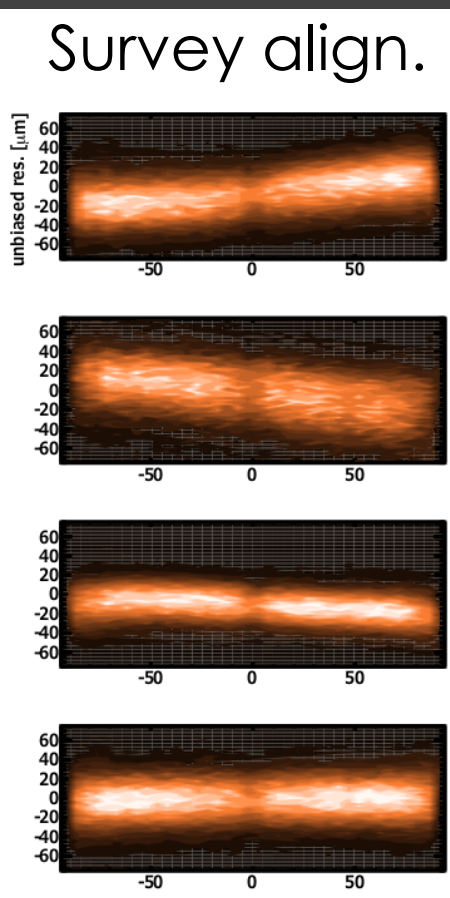

Track align.
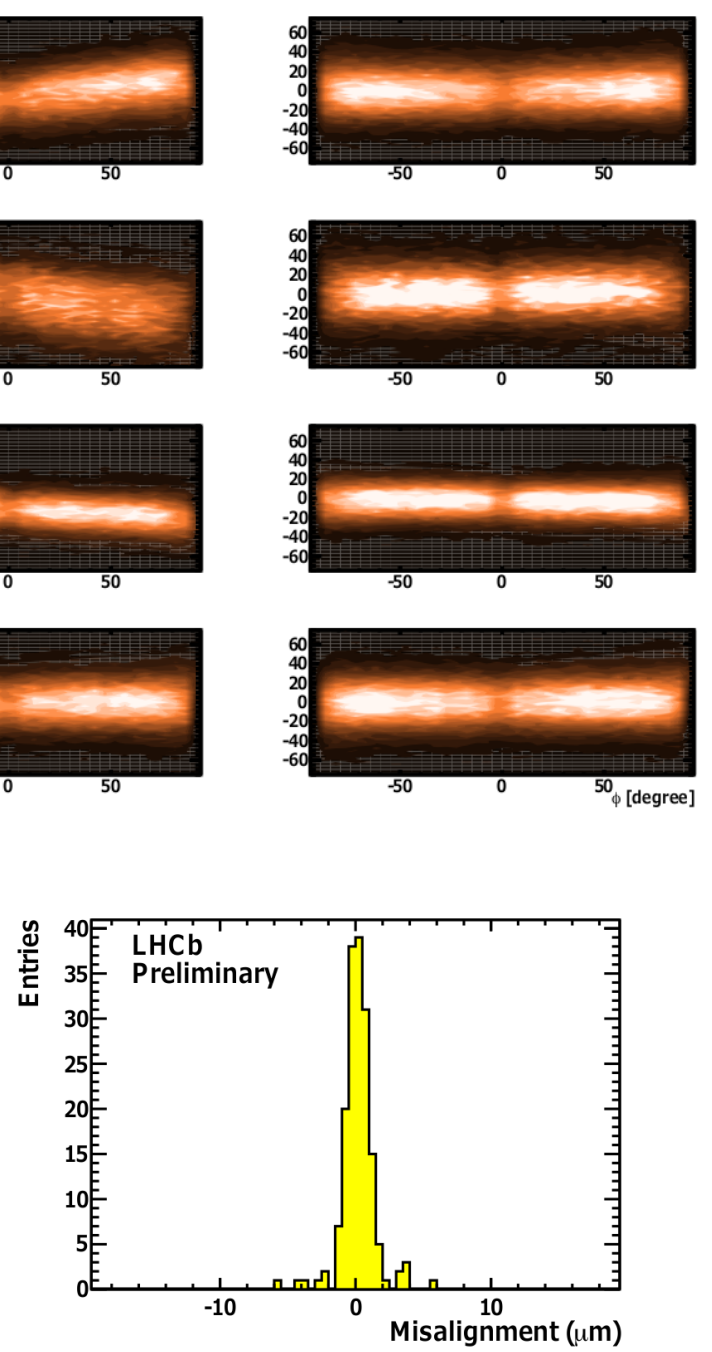


\section{Cluster finding efficiency}

- Determined by excluding a sensor in the pattern recognition, and interpolating tracks

to this sensor. Tracks are required to have:

- hits in both the $\mathrm{R}$ and $\Phi$ sensors in the 2+2 modules before/after the sensor under test.

a hits in at least six modules.

- These requirements place some restrictions on the region and sensors that can be probed.

- Track quality selection cuts are applied to remove fake.

- The efficiency for finding a cluster depends on the applied bias voltage.

CFE $=99.51 \pm 0.02 \%$ [all channels]

CFE $=99.98 \pm 0.02 \%$ [bad strips excluded $]$

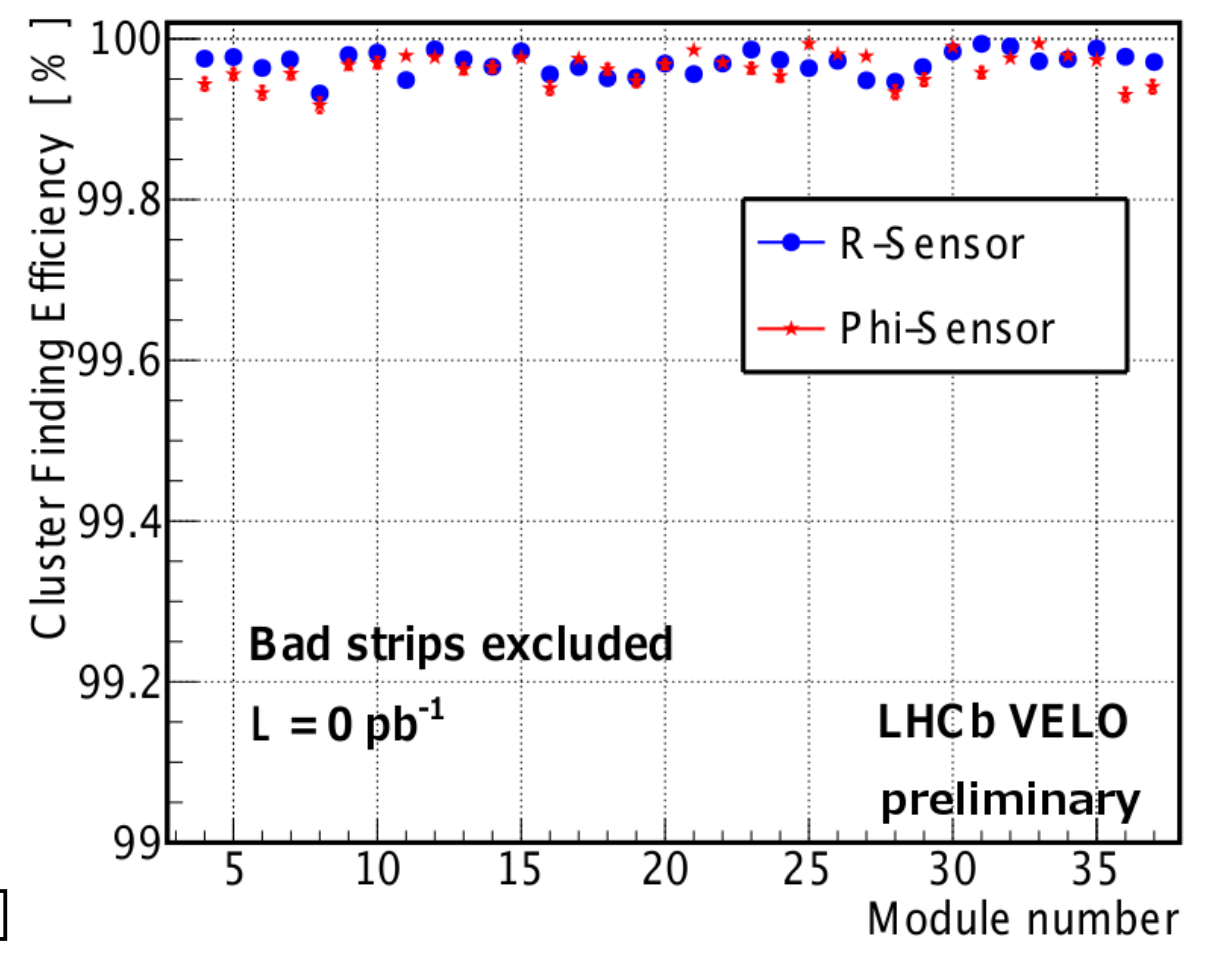




\section{Signal, Noise and S/N}
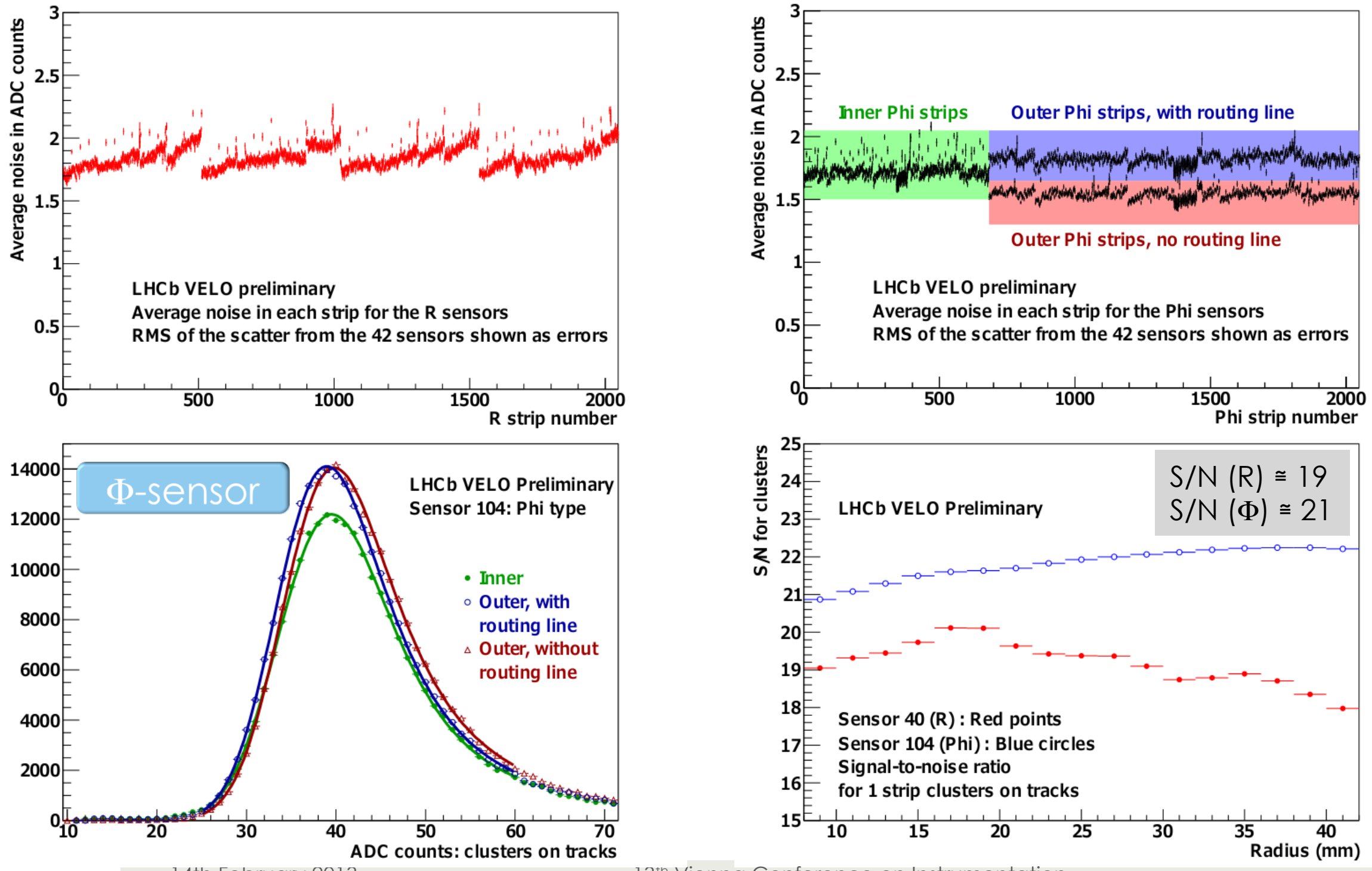


\section{Single hit resolution}

- Determined from the hit residuals.

- Charge sharing algorithm to improve resolution.

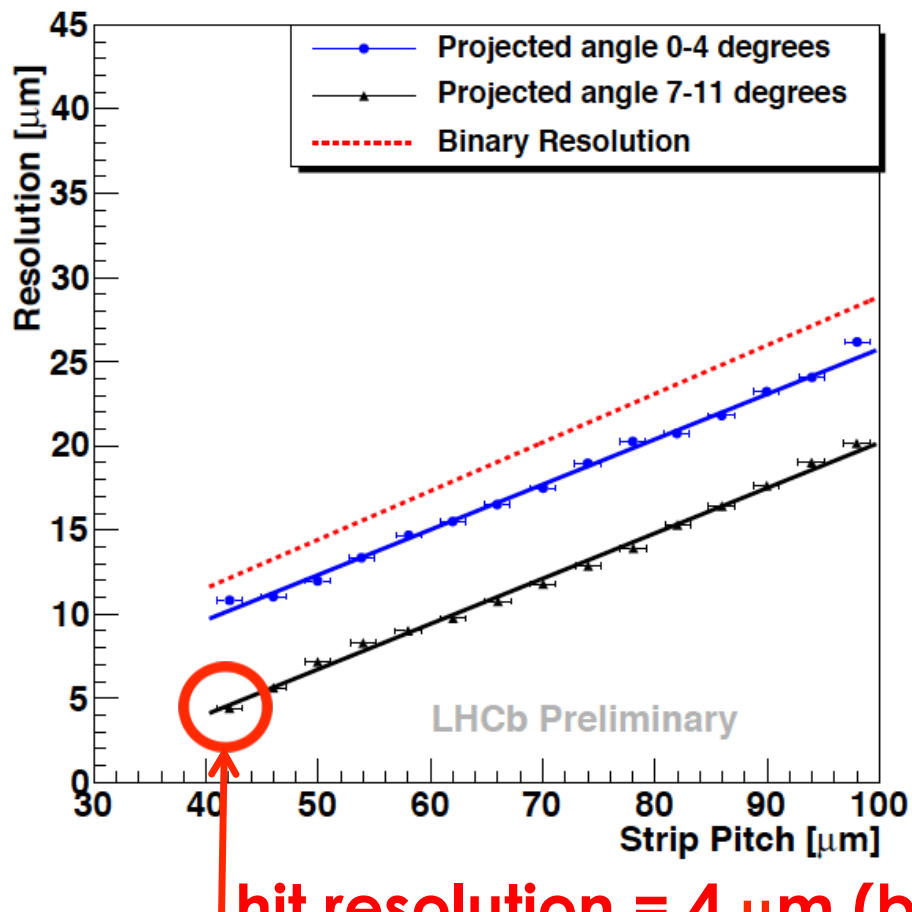

hit resolution = 4 um (best @ LHC!)
- Linear dependence on the strip pitch in projected angle bins.

- Significantly better resolution obtained for larger projected angles.

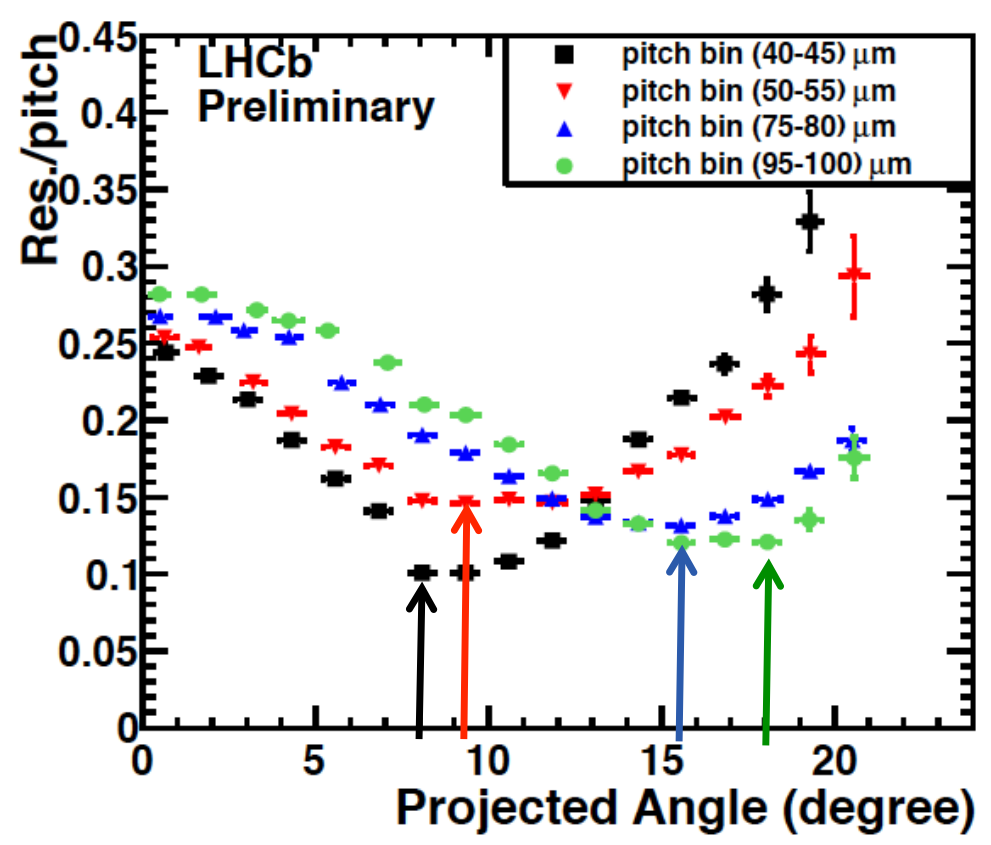




\section{Comparison with model}

- Comparison with Hamburg model.

- Good agreement at low and high fluences.

- Discrepancy around type inversion because of finite charge collection time.

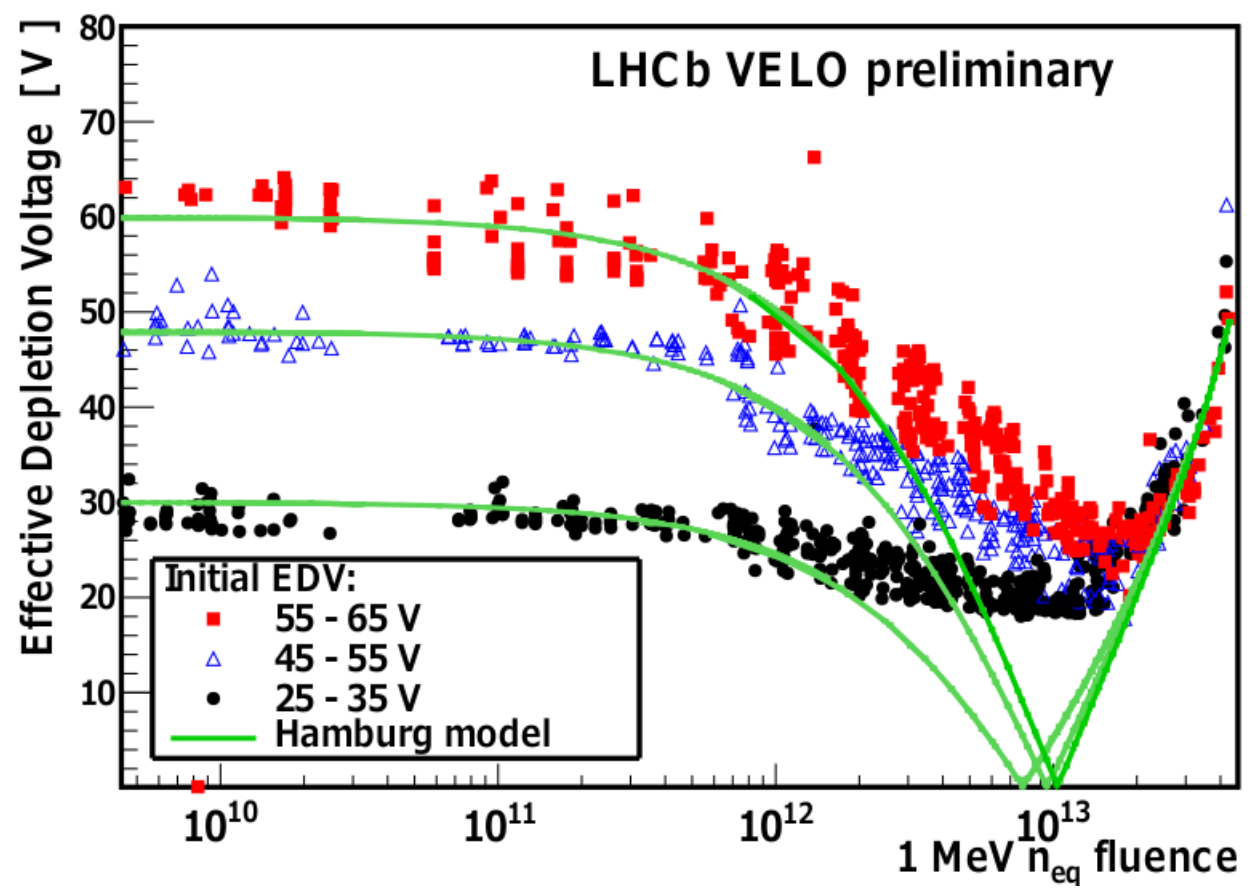




\section{IP \& PV resolution}

\section{$\mathbb{P}_{\mathrm{x}}$ Resolution Vs $1 / \mathrm{p}_{\mathrm{T}}$}

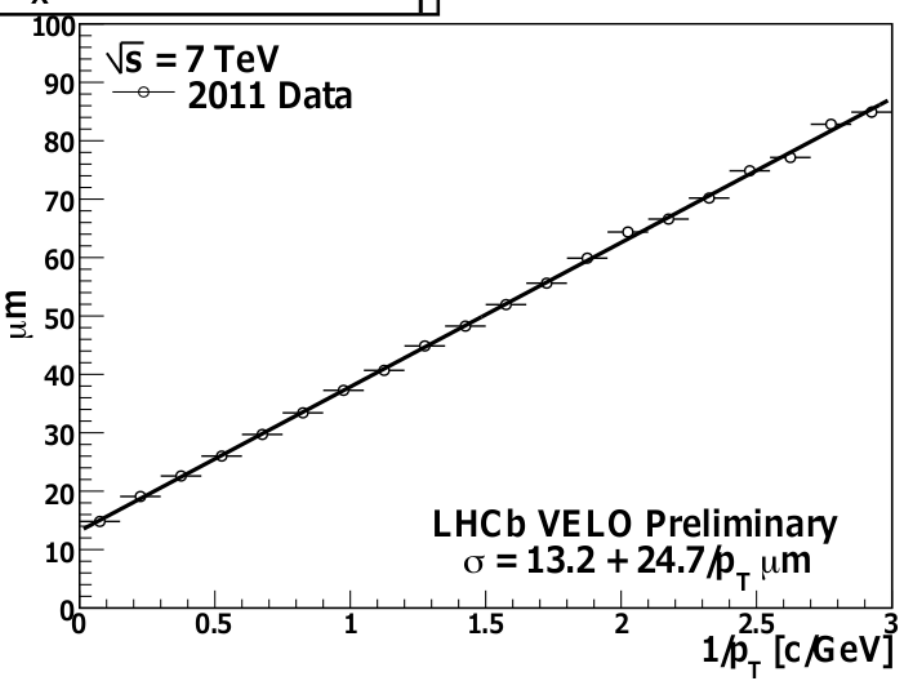

\section{$\mathbb{P}_{\mathrm{Y}}$ Resolution Vs $1 / \mathbb{p}_{\mathrm{T}}$}

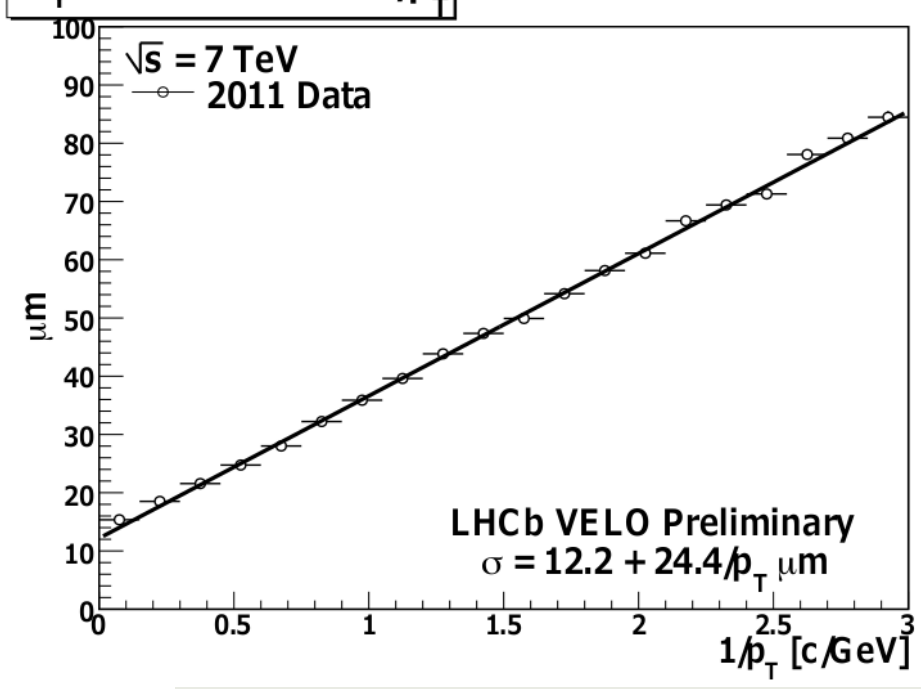

14th February 2013
$\mathrm{X}$ and $\mathrm{Y}$ resolution - offline, exactly $1 \mathrm{PV}$

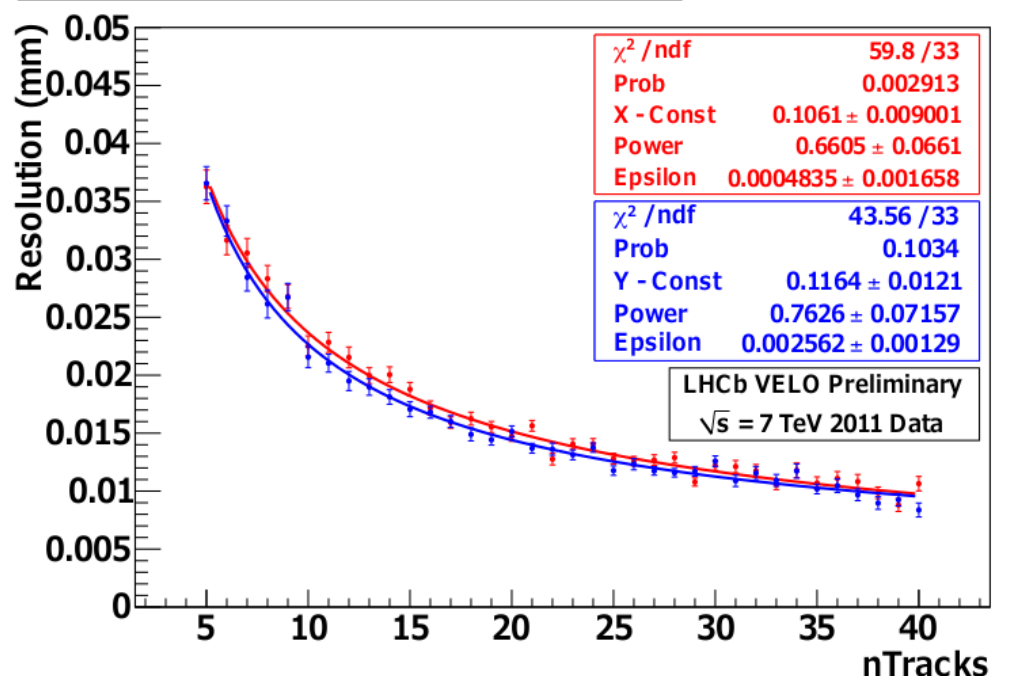

\section{$\mathrm{Z}$ resolution - offline, exactly $1 \mathrm{PV}$}

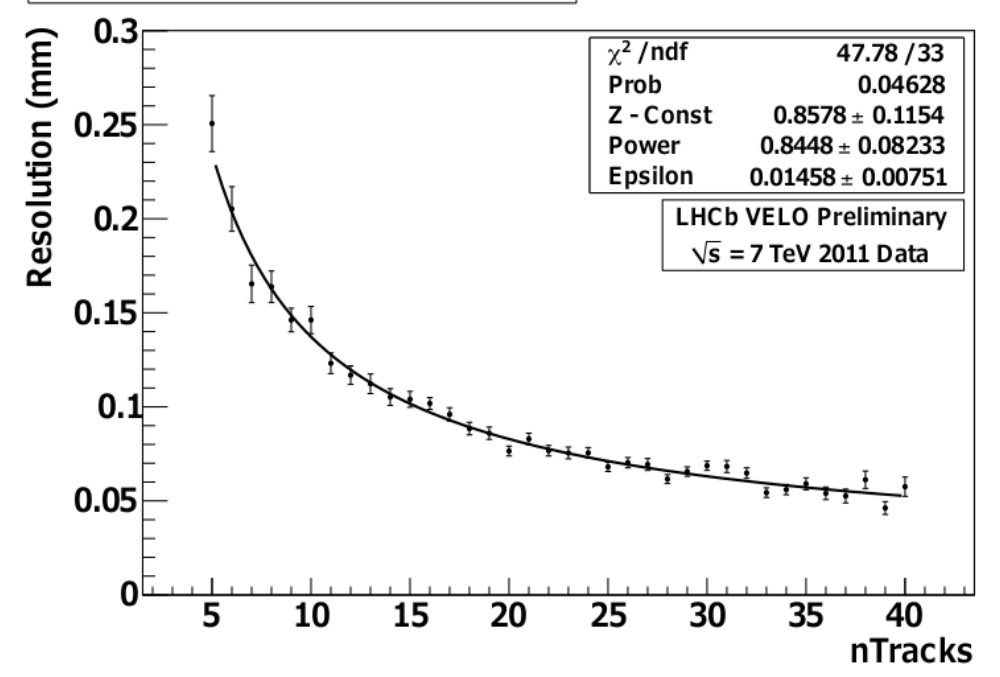




\section{Testbeam}

- Timepix telescope

- Designed for the LHCb upgrade

- Composed by 9 Timepix assemblies (with $300 \mu \mathrm{m}$ thick sensors)

- DUT can be moved/rotated and cooled

- Resolution at DUT plane $<2 \mu \mathrm{m}$

- Focus on:

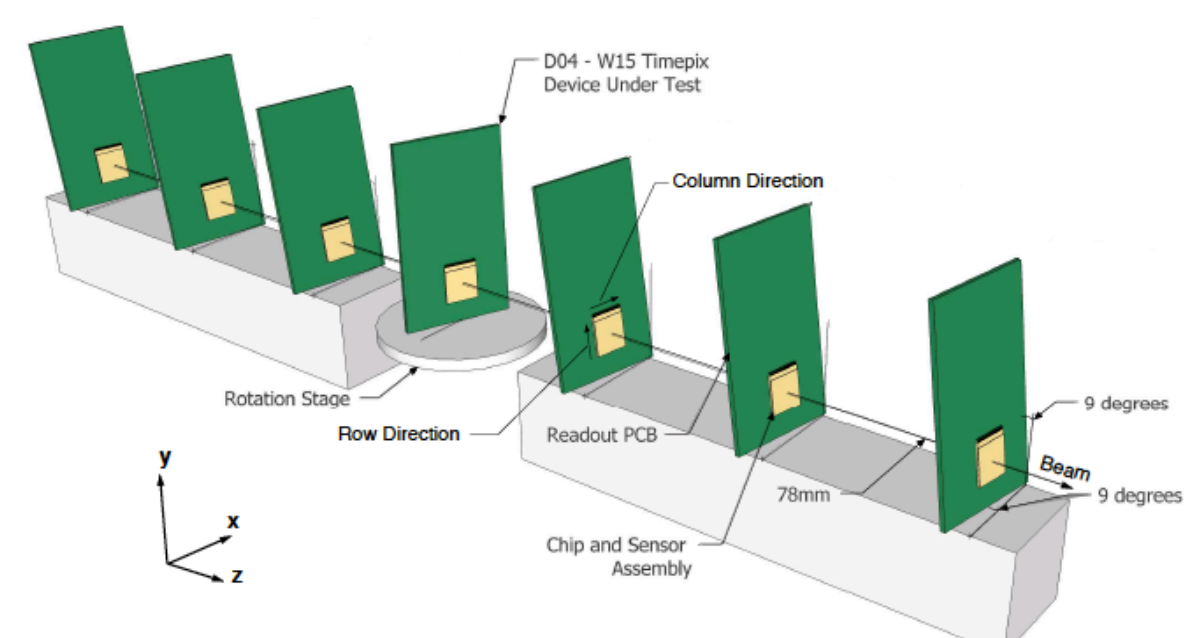

- sensor performance after irradiation (Meaıpıxs)

- evaluation of guard-rings, edge efficiency

- prototype strip module
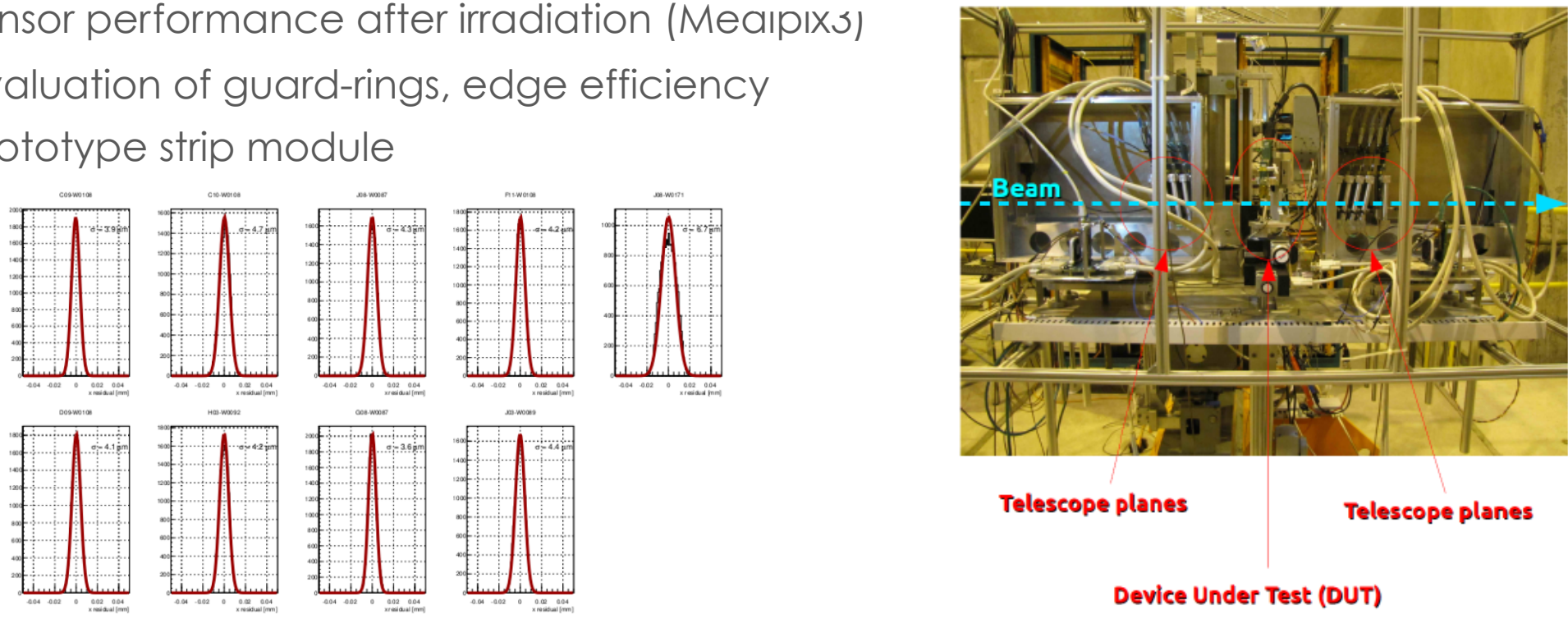

Telescope planes

Telescope planes

Device Under Test (DUT) 


\section{Data rates}

- Occupancy is "low" but the detector is fully read every 25 ns.

- Strips read out ZS data for each FE chip.

- Strip design compensates occupancy with shorter strips.

- Pixels summarize information from a $4 \times 4$ super pixel and time stamp the hits $\rightarrow 30 \%$ reduction on the rate.

- Intelligent column to readout data from hot to cold area

- Back end electronics must cope with a huge amount of data:

- TELL40 (upgrade of TELL1, current DAQ board) receives and builds events using FPGAs.

- All the information is assembled and passed on to computing farm, stripping down redundant data.

- Further processing and full reconstruction in the trigger farm.
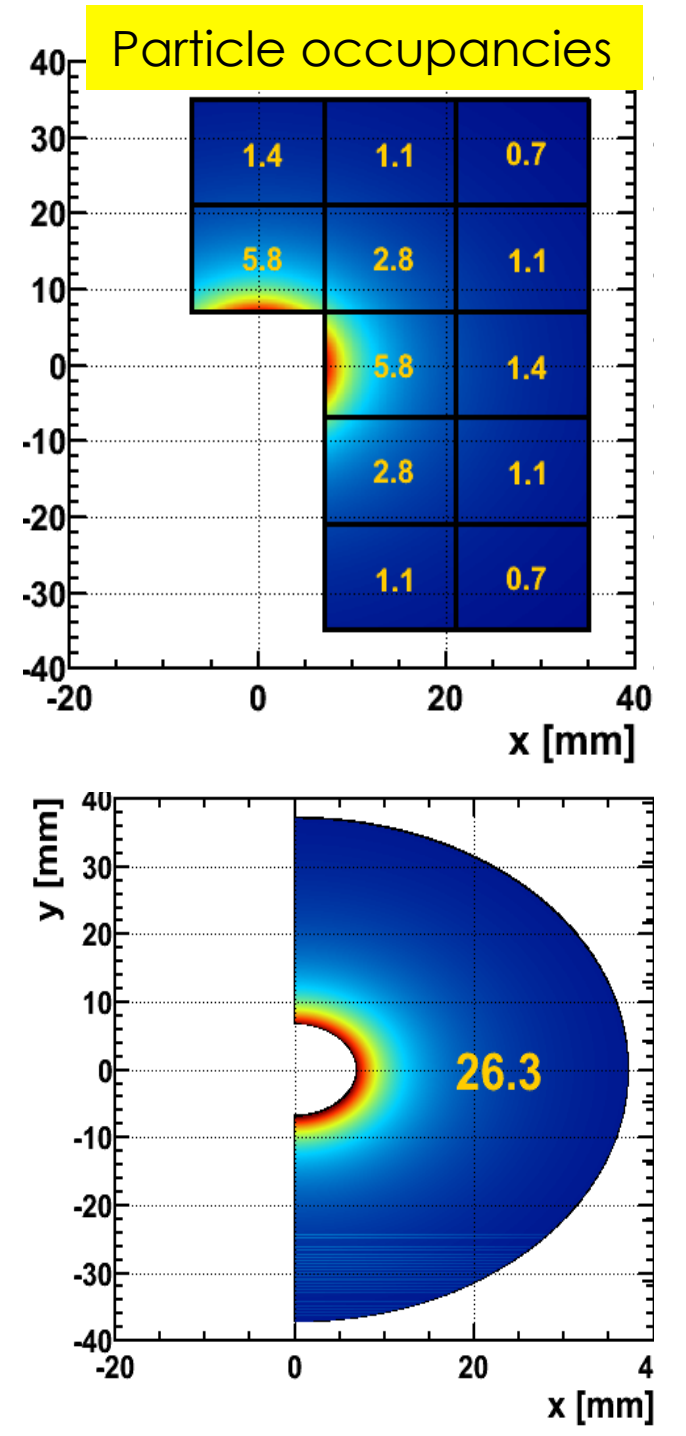